\title{
Polarization singularities in paraxial vector fields: morphology and statistics
}

\author{
M.R. Dennis \\ H.H. Wills Physics Laboratory, Tyndall Avenue, Bristol BS8 1TL, UK
}

Received 14 June 2002; received in revised form 3 September 2002; accepted 14 October 2002

\begin{abstract}
Polarization patterns in the transverse plane generically contain singularities: points of circular polarization $(\mathrm{C}$ points), lines of linear polarization (L lines), instantaneous zeros (disclinations) and component zeros. We investigate the geometry of ellipse fields at these singularities, using the Stokes parameters and others to characterize the singular geometry and morphology. Comparison is made with analogous structures on random surfaces, namely umbilic points and parabolic lines. The densities and correlations of the different types of polarization singularities are calculated in random polarization fields, and compared to the statistics of phase singularities and random surfaces.
\end{abstract}

(c) 2002 Elsevier Science B.V. All rights reserved..

PACS: 42.25.Ja; 03.65.Vf; 02.50; 42.30.Ms

Keywords: Polarization; C points; Phase singularities; Stokes parameters; Gaussian randomness

\section{Introduction}

Polarization patterns in paraxial optical fields are geometrically complicated: the electric field vector at each point is moving, and for monochromatic fields, sweeps out an ellipse. The shape, size and orientation of the ellipse varies with position in the transverse plane, and the most obvious features of the overall pattern are polarization singularities: places (usually points) where the ellipse is circular ( $\mathrm{C}$ points), curves along which the polarization is linear ( $\mathrm{L}$ lines, or $\mathrm{s}$ lines), and points where the instantaneous vector vanishes (disclinations). These objects were appreciated as generic features of paraxial polarization fields by
Nye [1-3], and have been the subject of much subsequent research, in theoretical, numerical and experimental aspects [4-16]. They are the vector analog of phase singularities (zeros, wave dislocations, optical vortices) in scalar fields [17-19]; the geometry and topology of vector fields is richer, and so are the singular structures.

The purpose of the present paper is twofold. Firstly, to present the mathematics of polarization singularities in order to make their connection with phase singularities clear, and to emphasize the connections between the different kinds of singularities. Secondly, to present the calculations of polarization singularity densities in random isotropic paraxial vector wavefields, thereby 
complementing the corresponding description and statistics of phase singularities [19-24] and polarization singularities in nonparaxial three-dimensional fields [7]. The random wave model is a useful means to examine some of the properties of singularities 'in the wild', that is, the generic features of the singularities. The singular structures arise naturally out of interference patterns, and are not due to any special symmetries. Attention will be restricted to monochromatic fields, and to the two-dimensional spatial structure in the transverse plane alone.

In addition to paraxial polarization patterns, two-dimensional fields of ellipses are found in the curvature fields on surfaces. That is, if $f$ is a real, smooth function in the $x, y$ plane, the gaussian curvature at a point in the plane is geometrically related to an ellipse. Places (generically points) where the curvature ellipse is circular are called umbilic points [25,26], and places (generically lines) where the gaussian curvature is zero (the curvature ellipse is linear) is linear are called parabolic lines [26]; these places are important for focusing when the surface represents the surface of a lens, mirror or screen, and they organize the caustics arising from geometrical optics [3]. Geometric properties of random surfaces were previously studied [25,27-29] mainly for this reason. In a sense, the present research applies these studies of geometric randomness to a different physical level: previous studies were on the geometric properties of surfaces as lenses, and their aim was to characterize the focusing of rays from them; the present work aims to do this for the morphology of the polarization wave patterns themselves. However, the calculations bear strong similarities to their random surface counterparts, as will be discussed.

Fields of ellipses also occur in oceanography, since the (changing) tidal current vector sweeps out an ellipse in time, so the ocean tidal current may be represented by a two-dimensional field of phased ellipses (for instance, see the figures of [30]), which generically has $\mathrm{C}$ points, L lines, and disclination points. The locations and nature of the $\mathrm{C}$ points in the North Sea were discussed in [8]. The morphology described in the present work applies to tidal singularities, although the statis- tics, which rely on an isotropic linear random wave model, do not. There are also similarities to the general flow fields studied in [31]. Geometrically close to $\mathrm{C}$ points are line field singularities, which occur physically as defects in plane confined liquid crystals [32], morphological features of fingerprint patterns [33], and in partial polarization patterns arising from scattering in the atmosphere from astronomical sources [34], and from sunlight $[35,36]$.

The most important tool in the study of any type of wave pattern singularity are phase singularities, that is, the zeros of a complex scalar field $\psi=\psi(x, y)=\psi(\mathbf{R})$ (we only consider fields in two dimensions). If $\psi$ represents an optical scalar field, the phase singularities are wave dislocations (optical vortices). Writing $\psi=\xi+\mathrm{i} \eta=\rho \exp (\mathrm{i} \chi)$ (where $\rho \geqslant 0, \chi, \xi, \eta$ are real), the phase singularities are places where the real and imaginary zero contours cross, and at these places the amplitude $\rho$ vanishes and the phase $\chi$ is indeterminate (singular). Phase singularities generically occur at points, and all equiphase lines (lines of constant $\chi$ ) meet at them. Around the singularity, the phase must change by $2 \pi s$, where $s$ is an integer, positive, negative or zero. $s$ is called the dislocation strength (or topological charge) of the singularity; the strength is positive if the phase increases in an anticlockwise sense, and decreases otherwise. Therefore, around a closed curve $\mathscr{L}$ which does not cross a zero of $\psi$, the line integral

$\oint_{\mathscr{L}} \mathrm{d} \chi=\oint \mathrm{d} \mathbf{R} \cdot \nabla \chi=2 \pi s$

gives the total dislocation strength enclosed by $\mathscr{L}$. Generically, phase singularities have strength \pm 1 , but more than one singularity may be enclosed by $\mathscr{L}$. For strength \pm 1 singularities, the sign of $s$ is given by the sign of the function $D[19,20]$,

$D=\xi_{x} \eta_{y}-\eta_{x} \xi_{y}$.

The foregoing discussion does not require $\psi$ to be a wavefield itself.

The plan of the paper is as follows: Section 2 provides a mathematical background to the relevant ellipse geometry of polarization singularities; it may be largely omitted by the reader familiar with this material. In Section 3, the various ways 
of describing $\mathrm{C}$ points and their structure are given, realized as phase singularities of different scalar fields. Section 4 follows with a description of $\mathrm{L}$ lines and relative singularities (component zeros, disclinations) found on them. The statistical densities of $\mathrm{C}$ points and $\mathrm{L}$ lines are computed in Appendix $\mathrm{B}$, using the isotropic random wave model formulated in Appendix A; the results are quoted and described in Section 5.

The main results of this paper are:

- The introduction of the phase sphere and associated phase parameters (Eq. (18)) as a descriptor of polarization ellipses complementary to the Poincaré sphere and Stokes parameters.

- The expressions $D_{\mathrm{L}}, D_{\mathrm{C}}$ (Eqs. (29) and (30)), giving the line and contour classification types of $\mathrm{C}$ point in terms of Stokes parameters.

- Example (31), generalizing the $\mathrm{C}$ point example of [8] to include all possible morphologies of $\mathrm{C}$ points (including the contour classification).

- Expression (40), which proves the main result of [15] in a simple way.

- The statistical results of Section 5, which gives the density (Eq. (41)) and correlation functions (Eqs. (46) and (47)) of $\mathrm{C}$ points in isotropic random fields, related to the corresponding dislocation statistics described in [19]. The Venn diagram of different fractions of types of $\mathrm{C}$ point is derived (Fig. 9), and compared to the corresponding diagram for umbilic points derived in [25]. The density of $\mathrm{L}$ lines is also given (Eq. (54)), and their statistical geometry and percolation properties are briefly compared to the curvature properties of random surfaces.

\section{Polarization ellipse morphology}

This section is concerned with the mathematical formalism of paraxial polarization, including the Stokes parameters and Poincaré sphere. Much of this description, with the exception of the phase sphere at the end of the section, can be found in standard textbooks, such as [37]. We are interested in the geometric structure of a two-dimensional complex vector wavefield $\mathbf{E}=\mathbf{E}(x, y)=\mathbf{E}(\mathbf{R})$, representing the electric field in the transverse $x, y$ plane of a paraxial wavefield (i.e., $\mathbf{E}$ is the Jones vector at $\mathbf{R}$ ). $\mathbf{E}$ may be rewritten in terms of cartesian components, or real and imaginary parts:

$\mathbf{E}=\left(E_{x}, E_{y}\right)=\mathbf{p}+\mathbf{i q}$.

The physical quantities $\mathbf{E}, \mathbf{p}, \mathbf{q}$ (and others) are taken to be smooth functions of position, although for brevity $\mathbf{R}$ dependence is usually not written explicitly. Derivatives will be represented by subscripts after scalars (as in Eq. (2)), and by commas after vector components (e.g., $\partial_{\mu} E_{\lambda}=E_{\lambda, \mu}$ ). The modulus of a real vector is denoted by italics, for instance $|\mathbf{V}|=V$.

The wave is monochromatic, and its time dependence is given by a phase factor $\exp (-\mathrm{i} \omega t)$, where $\omega$ is the unique angular frequency associated with the field. The real physical disturbance is

$\mathbf{E}_{\chi}^{\mathrm{Re}}=\operatorname{Re} \mathbf{E} \exp (-\mathrm{i} \chi)=\mathbf{p} \cos \chi+\mathbf{q} \sin \chi$,

where $\chi=\omega t$. Instead of explicitly treating time dependence of the field, $\mathbf{E}$ is dependent on the abstract phase $\chi$. Elementary linear algebra shows that $\mathbf{E}_{\chi}^{\mathrm{Re}}$ sweeps out an ellipse from its centre, as $\chi$ increases. The orthogonal semiaxes of the ellipse are denoted by $\mathbf{p}_{0}, \mathbf{q}_{0}$, with $p_{0} \geqslant q_{0}$. If $\mathbf{E}_{\chi}^{\mathrm{Re}}$ rotates anticlockwise as $\chi$ increases then $\mathbf{E}$ is said to be right-handed $(\mathrm{RH})$, if clockwise, left-handed $(\mathrm{LH})$. If $\mathbf{E}$ is $\mathrm{RH}$, the two-dimensional cross product $\operatorname{Im} \mathbf{E}^{*} \times \mathbf{E}=2 \mathbf{p} \times \mathbf{q}$ is positive, and if $\mathrm{LH}$, it is negative.

The cartesian components $E_{x}, E_{y}$ are complex scalar fields. Using a circular basis, $\mathbf{E}$ may be rewritten

$\mathbf{E}=\psi_{\mathrm{R}} \mathbf{e}_{\mathrm{R}}+\psi_{\mathrm{L}} \mathbf{e}_{\mathrm{L}}$,

where $\psi_{\mathrm{R}}, \psi_{\mathrm{L}}$ are the right- and left-handed circular components of $\mathbf{E}$, and the circular basis vectors, in cartesian coordinates, are defined

$\mathbf{e}_{\mathrm{R}}=\frac{1}{\sqrt{2}}\left(\begin{array}{l}1 \\ \mathrm{i}\end{array}\right), \quad \mathbf{e}_{\mathrm{L}}=\frac{1}{\sqrt{2}}\left(\begin{array}{c}1 \\ -\mathrm{i}\end{array}\right)$.

The phases of $\psi_{\mathrm{R}}, \psi_{\mathrm{L}}$ are defined as $\chi_{\mathrm{R}}, \chi_{\mathrm{L}}$ respectively. With these definitions, the right-handed component circulates in the anticlockwise direction. Note that some authors with this convention define the right-handed circular unit vector as $-\mathbf{e}_{\mathrm{R}}$.

In general, the real and imaginary parts $\mathbf{p}, \mathbf{q}$ are conjugate radii of the ellipse - the tangent to the ellipse at one is parallel to the other. There is 
therefore a special phase $\chi_{0}$ such that the real and imaginary parts of the resulting vector $\mathbf{E} \exp \left(-\mathrm{i} \chi_{0}\right)$ are perpendicular, in the direction of the semiaxes

$$
\begin{aligned}
\mathbf{p}_{0}+\mathrm{iq} & =\mathbf{E} \exp \left(-\mathrm{i} \chi_{0}\right) \\
= & \left(\mathbf{p} \cos \chi_{0}+\mathbf{q} \sin \chi_{0}\right) \\
& +\mathrm{i}\left(\mathbf{q} \cos \chi_{0}-\mathbf{p} \sin \chi_{0}\right) .
\end{aligned}
$$

Since the real and imaginary parts of (7) are orthogonal, it is possible to show that

$$
\begin{aligned}
\chi_{0} & =\frac{1}{2} \arctan \frac{2 \mathbf{p} \cdot \mathbf{q}}{p^{2}-q^{2}}=\frac{\chi_{\mathrm{R}}+\chi_{\mathrm{L}}}{2} \\
& =\frac{1}{2} \arg \psi_{\mathrm{R}} \psi_{\mathrm{L}},
\end{aligned}
$$

which is only defined modulo $\pi$. This phase was denoted by $\varepsilon$ in [37] and [1]. Nye [1] called $\chi_{0}$ the 'phase of the vibration', but we use the more concise term rectifying phase, because it rectifies (makes right-angled) the real and imaginary parts $\mathbf{p}, \mathbf{q}$.

Geometrically, the orientation of the ellipse is specified by the angle $\gamma_{0}$ between the major semiaxis $\mathbf{p}_{0}$ and the $x$-axis

$\gamma_{0}=\arctan \frac{p_{0 y}}{p_{0 x}}$.

Using Eqs. (7) and (8), this may be written solely in terms of the components of $\mathbf{p}, \mathbf{q}$, where, after simplification

$$
\begin{aligned}
\gamma_{0} & =\frac{1}{2} \arctan \frac{2\left(p_{x} p_{y}+q_{x} q_{y}\right)}{p_{x}^{2}+q_{x}^{2}-p_{y}^{2}-q_{y}^{2}}=\frac{\chi_{\mathrm{L}}-\chi_{\mathrm{R}}}{2} \\
& =\frac{1}{2} \arg \psi_{\mathrm{R}}^{*} \psi_{\mathrm{L}} .
\end{aligned}
$$

This expression is similar to expression (8) for $\chi_{0}$, and is also only defined modulo $\pi$. The relation between the geometry of the polarization ellipse and the parameters defined here are shown in Fig. 1.

The polarization matrix (coherence matrix, or Stokes matrix) $\mathscr{M}_{S}$ is a $2 \times 2$ hermitian matrix defined by its components

$$
\mathscr{M}_{S \lambda \mu}=E_{\lambda} E_{\mu}^{*}, \quad \lambda, \mu=x, y,
$$

and its real part $\mathscr{M} \equiv \operatorname{Re} \mathscr{M}_{S}$ is a positive definite symmetric matrix whose eigenvectors are in the directions $\mathbf{p}_{0}, \mathbf{q}_{0}$, and whose eigenvalues are $p_{0}^{2}$ and $q_{0}^{2}$. In fact, $\mathscr{M}^{-1}$, if it exists, is the quadratic form

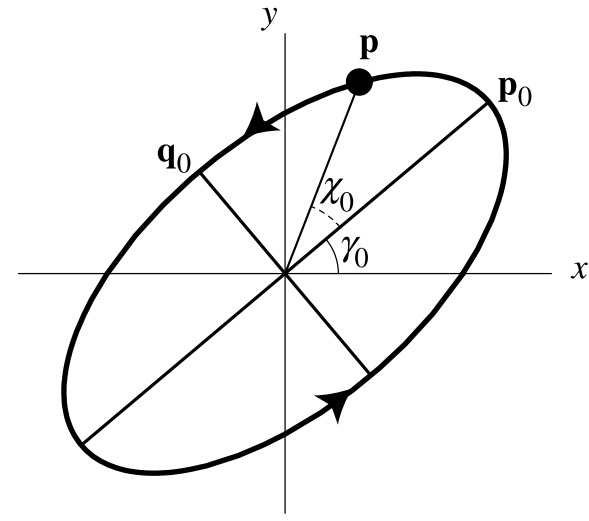

Fig. 1. Ellipse geometry. The major axis $\mathbf{p}_{0}$ is at angle $\gamma_{0}$ with the $x$-axis. The real part $\mathbf{p}$ is displaced from $\mathbf{p}_{0}$ by a phase $\chi_{0}$, as in Eq. (7). The minor axis is labelled $\mathbf{q}_{0}$, and the ellipse is righthanded (RH).

matrix for the ellipse. The four components of the complex polarization matrix with respect to the usual Pauli spin matrices are known as the Stokes parameters $S_{0}, S_{1}, S_{2}, S_{3}$, i.e.,

$\mathscr{M}_{S}=\frac{1}{2}\left(\begin{array}{cc}S_{0}+S_{1} & S_{2}-\mathrm{i} S_{3} \\ S_{2}+\mathrm{i} S_{3} & S_{0}-S_{1}\end{array}\right)$,

and they are given by

$$
\begin{aligned}
S_{0} & =\left|\psi_{\mathrm{R}}\right|^{2}+\left|\psi_{\mathrm{L}}\right|^{2} \\
& =\left|E_{x}\right|^{2}+\left|E_{y}\right|^{2}=p_{x}^{2}+p_{y}^{2}+q_{x}^{2}+q_{y}^{2}, \\
S_{1} & =2 \operatorname{Re}\left(\psi_{\mathrm{R}}^{*} \psi_{\mathrm{L}}\right)=\left|E_{x}\right|^{2}-\left|E_{y}\right|^{2} \\
& =p_{x}^{2}+q_{x}^{2}-p_{y}^{2}-q_{y}^{2}, \\
S_{2} & =2 \operatorname{Im}\left(\psi_{\mathrm{R}}^{*} \psi_{\mathrm{L}}\right)=2 \operatorname{Re}\left(E_{x}^{*} E_{y}\right) \\
& =2\left(p_{x} p_{y}+q_{x} q_{y}\right), \\
S_{3} & =\left|\psi_{\mathrm{R}}\right|^{2}-\left|\psi_{\mathrm{L}}\right|^{2}=2 \operatorname{Im}\left(E_{x}^{*} E_{y}\right) \\
& =2\left(p_{x} q_{y}-p_{y} q_{x}\right) .
\end{aligned}
$$

The expressions for $S_{i}(i=1,2,3)$ in the circular components $\psi_{\mathrm{R}}, \psi_{\mathrm{L}}$ are evidently a cyclic permutation of the expressions in cartesian components $E_{x}, E_{y}$; this is because the Pauli matrices (which give the Stokes parameters) are permuted by the transformation between the cartesian and circular basis (6), as may be easily checked. Physically, $S_{0}$ represents the intensity $|\mathbf{E}|^{2}$ of the field, and $\pi\left|S_{3}\right| / 2$ is the area of the polarization ellipse; the sign of $S_{3}$ gives the handedness of the ellipse, + for 
$\mathrm{RH},-$ for $\mathrm{LH}$ (it is equal to $\mathbf{p} \times \mathbf{q}$ ). By (10) and (13), $\tan 2 \gamma_{0}=S_{2} / S_{1}$. The Stokes parameters are independent of the rectifying phase $\chi_{0}$.

The Stokes parameters, as defined, satisfy

$S_{0}^{2}=S_{1}^{2}+S_{2}^{2}+S_{3}^{2}$,

confirming that the polarization is pure, and they can be normalized

$s_{i}=S_{i} / S_{0}, \quad i=1,2,3$.

The unit vector $\left(s_{1}, s_{2}, s_{3}\right)$ is called the (normalized) Stokes vector, and its direction in abstract Stokes space describe the orientation and shape of the polarization ellipse. The possible states of polarization are therefore parameterized by positions on the unit sphere, called the Poincare sphere. All possible polarization ellipses (up to size, given by $S_{0}$, and rectifying phase $\chi_{0}$ ), are parameterized by position on the Poincaré sphere, given by the spherical polar angles $\alpha, \beta$. The geometry of the Poincare sphere is shown in Fig. 2. The orientation $\gamma_{0}$ is governed by the azimuthal angle

$\beta=2 \gamma_{0}$,

and the polar angle $\alpha=\arccos s_{3}$ is related to the eccentricity $\varepsilon$ of the ellipse, whose square is found to be given by the following expressions:

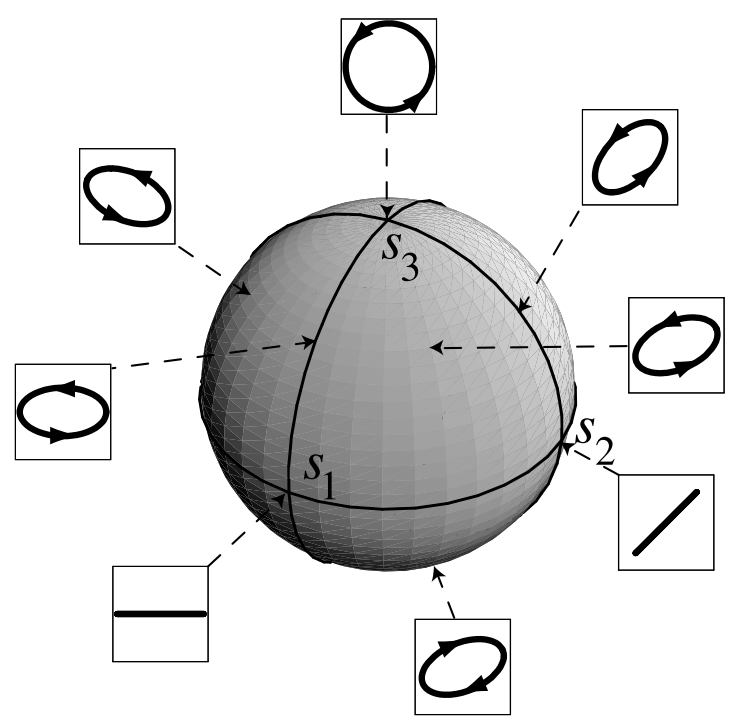

Fig. 2. Depiction of the Poincare sphere, with cartesian axes $\left(s_{1}, s_{2}, s_{3}\right)$ indicating the Stokes parameters for certain ellipses.

$$
\begin{aligned}
\varepsilon^{2} & =1-\frac{q_{0}^{2}}{p_{0}^{2}}=\frac{4\left|\psi_{\mathrm{R}}\right|\left|\psi_{\mathrm{L}}\right|}{\left(\left|\psi_{\mathrm{R}}\right|+\left|\psi_{\mathrm{L}}\right|\right)^{2}} \\
& =\frac{4 \tan \alpha / 2}{(1+\tan \alpha / 2)^{2}} \\
& =\frac{2}{S_{3}^{2}}\left(S_{0}-\sqrt{S_{0}^{2}-S_{3}^{2}}\right) \sqrt{S_{0}^{2}-S_{3}^{2}}
\end{aligned}
$$

The eccentricity therefore changes with latitude: at the poles, it is 0 (circular polarization), gradually increasing to 1 (linear polarization) on the equator. $\mathrm{RH}$ polarization is in the northern hemisphere $\left(S_{3}>0\right)$, LH in the southern $\left(S_{3}<0\right)$. The axis ratio, signed by handedness, is given by $\tan (\pi / 4-\alpha / 2)$.

The information about the rectifying phase $\chi_{0}$ is lost in the Stokes parameters (13), which only contain information about intensity, handedness, eccentricity and orientation (which are measurable quantities). It is therefore possible to define a sphere equivalent to the Poincaré sphere, but using the rectifying phase $\chi_{0}$ rather than orientation angle $\gamma_{0}$ as azimuth [38]. The cartesian coordinates on the phase sphere are given by the phase parameters (cf. Eq. (13))

$$
\begin{aligned}
& T_{0}=|\mathbf{E}|^{2}=S_{0}, \\
& T_{1}=2 \operatorname{Re}\left(\psi_{\mathrm{R}} \psi_{\mathrm{L}}\right)=p^{2}-q^{2}, \\
& T_{2}=2 \operatorname{Im}\left(\psi_{\mathrm{R}} \psi_{\mathrm{L}}\right)=2(\mathbf{p} \cdot \mathbf{q}), \\
& T_{3}=\left|\psi_{\mathrm{R}}\right|^{2}-\left|\psi_{\mathrm{L}}\right|^{2}=S_{3} .
\end{aligned}
$$

The zeroth and third phase parameters are identical to the corresponding Stokes parameters, so the eccentricity dependence on latitude (17) is the same as on the Poincaré sphere. $T_{1}$ and $T_{2}$ are the same as the corresponding Stokes parameters except $\psi_{\mathrm{R}}$ is no longer conjugated, equivalent to exchanging $p_{y}$ and $q_{x}$. The azimuth $\arctan T_{2} / T_{1}$ is equal to $2 \chi_{0}$, from (8). Therefore, the phase (visualized as the position of $\mathbf{p}=\operatorname{Re} \mathbf{E}$ on the ellipse), varies with azimuth around the sphere.

It is worth noting that the geometry described above only applies to ellipse fields in two dimensions, when the ellipses are all confined to the plane. If the polarization field is nonparaxial, and the plane of the ellipse changes from point to point, the Poincaré sphere is no longer appropriate to describe the state, since the ellipse has no unique 
handedness. The correct polarization geometry can be found using the Majorana sphere [39,40], and this leads to some differences in the geometry of polarization singularities in paraxial and nonparaxial fields $[4,7,8]$.

\section{C points and their geometry}

The most important type of polarization singularities are $\mathrm{C}$ points [1], that is, places in the polarization field where the polarization is circular (either RH or LH). This generically happens at points because $\mathrm{C}$ singularities have codimension 2, that is, two real conditions on the field variables must be satisfied for the polarization to be circular. They may be viewed in several different ways:

- The real and imaginary vectors $\mathbf{p}, \mathbf{q}$ are of equal length and orthogonal. This pair of conditions holds for both the paraxial and nonparaxial case, and has been used as the definition of $\mathrm{C}$ points several times in the literature $[1,3,8,11]$. Note that this geometric condition is equivalent to the requirement on the phase parameters

$$
\left.\begin{array}{c}
T_{1}=p^{2}-q^{2}=0 \text { (equal length) } \\
T_{2}=2 \mathbf{p} \cdot \mathbf{q}=0 \text { (orthogonal) } \\
\text { at a } \mathrm{C} \text { point. }
\end{array}\right\}
$$

The vanishing of these two parameters is equivalent to the statement that the rectifying phase $\chi_{0}$ is undefined (singular) at a $\mathrm{C}$ point, since $\mathbf{p}, \mathbf{q}$ are rectified for any phase.

- The Stokes parameters $S_{1}, S_{2}$ vanish at the C point. The two points of circular polarization on the Poincaré sphere lie at the north and south poles, where $S_{3}= \pm S_{0}$, and so $S_{1}=S_{2}=0$ by (14). The azimuthal angle $\beta$ is singular at the poles of a sphere, and this corresponds to the singularity of the orientation angle $\gamma_{0}$ : no unique semiaxes can be defined for a circle. This is a different singularity from that of $\chi_{0}$, although the two singularities always occur together, at the poles of the phase sphere and Poincaré sphere. The C point is RH if $S_{3}=S_{0}$, LH if $S_{3}=-S_{0}$.

- The real symmetric matrix $\mathscr{M}$ is degenerate (this is obvious from the above point and the defini- tion (12)). Degeneracies of real symmetric matrices are studied in more general contexts $[41,42]$, and all have codimension 2. The structure of these matrices around the singularity is called a diabolical point because the two eigenvalues locally have a double cone structure. This interpretation was used to define the umbilic points of a real function $f$ in [25,3], as degeneracies of its hessian matrix $\mathscr{H}=f_{\alpha \beta}$ $(\alpha, \beta=x, y)$.

- The simplest and possibly most instructive way of viewing $\mathrm{C}$ points is to consider the circular components $\psi_{\mathrm{R}}, \psi_{\mathrm{L}}$; a point of $\mathrm{RH}$ circular polarization occurs where $\psi_{\mathrm{L}}=0$, and equivalently LH circular polarization where $\psi_{\mathrm{R}}=0$ that is, C points are dislocations of $\psi_{\mathrm{R}}, \psi_{\mathrm{L}}$ $[1,3,5]$. The phases $\chi_{\mathrm{R}}, \chi_{\mathrm{L}}$ are therefore singular at the respective $C$ points, and by Eqs. (8), (10), $\chi_{0}$ and $\gamma_{0}$ are automatically both singular when either $\chi_{\mathrm{R}}$ or $\chi_{\mathrm{L}}$ is.

The above implies that the ellipse orientation $\gamma_{0}$ is undefined at a $\mathrm{C}$ point. As with phase singularities and vector fields, this implies that the line integral on a loop $\mathscr{L}$,

$\frac{1}{2 \pi} \oint_{\mathscr{L}} \mathrm{d} \mathbf{R} \cdot \nabla \gamma_{0}=I$,

is quantized as in (1), but in units of $1 / 2$ since $\gamma_{0}$ is only defined modulo $\pi$. This number $I$ is said to be the $\mathrm{C}$ point index, and is generically $\pm 1 / 2$. C points may be thought of as singularities in line fields (that is, fields of 'headless vectors' [32]); lines are brought back to themselves after half a full rotation, and this happens generically around a $\mathrm{C}$ point.

So far, we have seen that generic $\mathrm{C}$ points may be classified into four types, according to handedness $(\mathrm{RH}$ or $\mathrm{LH})$ and index $( \pm 1 / 2)$. A scalar field may be defined from the Stokes parameters [9-11]

$\sigma=S_{1}+\mathrm{i} S_{2}$.

This vanishes exactly at a $\mathrm{C}$ point (where $S_{1}=S_{2}=0$ ), and its phase is clearly

$\arg \sigma=\beta=2 \gamma_{0}$.

The signed strength of any phase singularity in $\sigma$ is therefore twice the index of the $\mathrm{C}$ point there. The 
sign of the index of a $\mathrm{C}$ point can therefore be found as sign $D_{\mathrm{I}}$, where $D_{\mathrm{I}}$ is defined

$D_{\mathrm{I}}=S_{1 x} S_{2 y}-S_{1 y} S_{2 x}$,

the $\sigma$ analog to Eq. (2). This agrees with the form stated in Appendix 3 of [31].

$\mathrm{C}$ points are also phase singularities in the circular components $\psi_{\mathrm{L}}, \psi_{\mathrm{R}}$, but how do the signs of these dislocations agree with the $\mathrm{C}$ point index? By Eq. (13),

$\sigma=\psi_{\mathrm{R}}^{*} \psi_{\mathrm{L}}$,

implying that the strength of the dislocation in $\psi_{\mathrm{L}}$, at a $\mathrm{RH} C$ point, has the same sign as the $\mathrm{C}$ point index there, but the strength of a dislocation of $\psi_{\mathrm{R}}$, at a $\mathrm{LH} \mathrm{C}$ point, is minus the sign of the $\mathrm{C}$ point index (this may also be seen using (10) [5]). $\sigma$ is therefore a complex scalar field which has phase singularities exactly at the $\mathrm{C}$ points, and the signs of its phase singularities are the same as the $\mathrm{C}$ point indices. However, just like the Stokes parameters themselves, this field is not itself a solution of any wave equation, and is quadratic in the field variables.

An alternative scalar with nodes at $\mathrm{C}$ points was used by $[7,8]$, and is defined as the scalar product of $\mathbf{E}$ with itself

$\varphi=\mathbf{E} \cdot \mathbf{E}$.

This vanishes when the polarization is circular, and from Eqs. (18),

$\varphi=T_{1}+\mathrm{i} T_{2}=\psi_{\mathrm{R}} \psi_{\mathrm{L}}$.

Its phase $\arg \varphi$ is clearly equal to $2 \chi_{0}$, and the moduli $|\varphi|,|\sigma|$ are equal

$|\varphi|^{2}=|\sigma|^{2}=S_{0}^{2}-S_{3}^{2}=\left(p_{0}^{2}-q_{0}^{2}\right)^{2}=p_{0}^{4} \varepsilon^{4}$.

Eq. (26) shows that the sign of a phase singularity in $\varphi$ is always the same as the dislocation strength in the circular component at that point, and therefore is opposite in sign to the $\mathrm{C}$ point index at a LHC point. The equiphase lines of $\varphi$ are lines of constant $\chi_{0}$; the lines $T_{1}=0$ and $T_{2}=0$, from (18), are respectively lines along which $\mathbf{p}$ and $\mathbf{q}$ have equal length, and are orthogonal. The equiphase lines of $\sigma$ are lines where the orientation angle $\varphi$ is constant, the $a$-lines discussed by [16]; we suggest the more evocative name isoclines for these lines. As complex scalar fields, $\sigma, \varphi, \psi_{\mathrm{R}}, \psi_{\mathrm{L}}$,

satisfy the myriad sign rules discussed by [22] Each of these phase functions has saddles and possibly maxima and minima, which are stationary points of the appropriate angle, as described by [16].

$\mathrm{C}$ points are singularities of their orientation angle $\gamma_{0}$, which represents an undirected line at each point; we therefore discuss the wider but morphologically close phenomenon of line singularities. Line fields can be classified according to their index, that is, the signed number of turns the line makes around the singularity in a righthanded sense, in units of $1 / 2$. The number of straight lines that terminate on the singularity is generically 3 or 1 . This is the line classification, and for index $-1 / 2$ singularities, it is always 3 ; such a singularity is called a star. If the index is $+1 / 2$, it may either be 1 or 3 , and the two morphologies are called lemon and monstar, respectively [25]. The three singularity types in line fields are shown in Fig. 3. The four polarization neutral points that occur from sunlight scattering in the atmosphere $[35,36]$, not described by the present theory since they involve partial polarization, are nevertheless generic index $+1 / 2$ line field singularities, and the polarization pattern around them is of lemon type (as can be seen from the figures in [36]).

In Section 5 the relative densities of lemon, monstar and star types of $\mathrm{C}$ point in gaussian random polarization fields are discussed, complementing the corresponding calculation [25] for umbilic points. In fact, it was in the context of umbilic points that this classification was originally recognized $[25,26]$, where the linefields are the principal curvature directions (and so are sin-

(a)

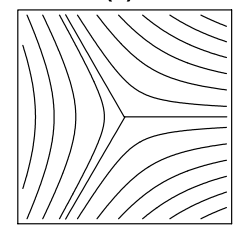

Fig. 3. The patterns around the three types of line singularity: (a) star; (b) lemon; (c) monstar. Adapted from [8], Fig. 6, courtesy of Michael Berry. 
gular at degeneracies of the hessian, as previously observed).

In order to distinguish between the line classification types, we need a quantity equivalent to $D_{\mathrm{I}}$ in Eq. (23) whose sign gives the type of line classification. The quantity defined in Eq. (6) of [25] is not appropriate for $\mathrm{C}$ points, because the derivatives of the components of $\mathscr{M}$ are more general than those of $\mathscr{H}$. However, the appropriate expression for $\mathrm{C}$ points may be derived as follows. The $\mathrm{C}$ point is translated to the origin, and $S_{1}, S_{2}$ in the expression $\gamma_{0}=\arctan \left(S_{2} / S_{1}\right) / 2$ are expanded to first order (at the $\mathrm{C}$ point) with polar coordinates $R, \theta$, with angle $\theta=\arctan (y / x)$. This expression is rearranged to a polynomial equation in $t=\tan \theta$,

$S_{2 y} t^{3}+\left(S_{2 x}+2 S_{1 y}\right) t^{2}+\left(2 S_{1 x}-S_{2 y}\right) t-S_{2 x}=0$,

whose solutions give the angles where $\theta=\gamma_{0}$, that is, the angles of the straight lines terminating at the singularity. The number of these solutions is given by the discriminant of the polynomial (28), which is (up to an unimportant numerical factor)

$$
\begin{aligned}
D_{\mathrm{L}}= & \left(\left(2 S_{1 y}+S_{2 x}\right)^{2}-3 S_{2 y}\left(2 S_{1 x}-S_{2 y}\right)\right) \\
& \times\left(\left(2 S_{1 x}-S_{2 y}\right)^{2}+3 S_{2 x}\left(2 S_{1 y}+S_{2 x}\right)\right) \\
& -\left(2 S_{1 x} S_{1 y}+S_{1 x} S_{2 x}-S_{1 y} S_{2 y}+4 S_{2 x} S_{2 y}\right)^{2} .
\end{aligned}
$$

The sign of the discriminant gives the number of roots -3 (star or monstar) if $D_{\mathrm{L}}>0,1$ (lemon) if $D_{\mathrm{L}}<0$. This expression is equivalent to that of [31], and Eq. (6) of [25] after the appropriate substitution for umbilic points.

There is an additional classification, the contour classification which specifies whether the singularity is elliptic or hyperbolic, according to whether the contour lines of the eigenvalues of $\mathscr{M}$ around the degeneracy are ellipses or hyperbolas. For umbilic points, this classification determines whether the resulting catastrophe of the normal rays is the elliptic or hyperbolic umbilic catastrophe [3]. It is not clear whether there is any correspondingly simple physical interpretation for this classification for $\mathrm{C}$ points. The function $D_{\mathrm{C}}$, whose sign determines the contour classification, was stated in [31] Appendix 3; in terms of Stokes parameters, it is proportional to

$$
\begin{aligned}
D_{\mathrm{C}}= & \left(S_{1 x} S_{2 y}-S_{1 y} S_{2 x}\right)^{2}-\left(S_{1 x} S_{0 y}-S_{1 y} S_{0 x}\right)^{2} \\
& -\left(S_{0 x} S_{2 y}-S_{0 y} S_{2 x}\right)^{2} .
\end{aligned}
$$

The point is elliptic if $D_{\mathrm{C}}>0$, and hyperbolic if $D_{\mathrm{C}}<0$. It may be readily checked that, at $\mathbf{R}_{0}, D_{\mathrm{C}}$ is the gaussian curvature of the surface locally defined $\operatorname{det}\left(\mathscr{M}(\mathbf{R})-\mathscr{M}\left(\mathbf{R}_{0}\right)\right)$; this surface - whose interpretation also holds for umbilic points - is the product of the differences of the two eigenvalues of $\mathscr{M}$ from their degenerate value. Two C points from a random polarization pattern are shown in Fig. 4, with their line and contour types indicated.

An instructive example is the field

$\mathbf{E}_{\mathrm{ex}}=(1+\mathrm{i}(a x+b y), \mathrm{i}(1-x))$,

where $a, b$ are real. There is a $\mathrm{RH} \mathrm{C}$ point at the origin, and its classification parameters $D_{\mathrm{I}}, D_{\mathrm{L}}, D_{\mathrm{C}}$ are

$D_{\mathrm{I}}=4 b(1+a)$,

$D_{\mathrm{L}}=-16 b(2+2 a-b)^{3}$,

$D_{\mathrm{C}}=64 a b^{2}$.

The form with $a=0$ was given in [8] Eq. (17). All six morphological types are clearly possible (elliptic or hyperbolic, lemon, star or monstar) with appropriate choices of $a$ and $b$, as shown in Fig. 5. The conjugate field $\mathbf{E}_{\mathrm{ex}}^{*}$ to (31) has a LH C point at the origin (the classification parameters in (34) are (a)

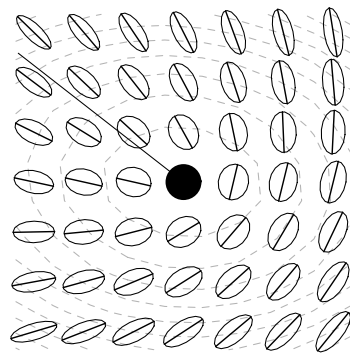

(b)

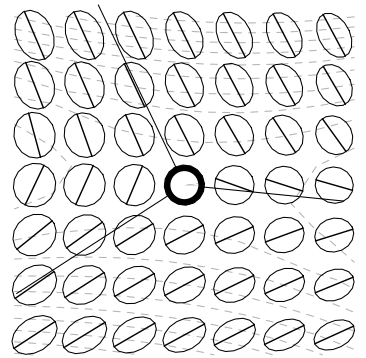

Fig. 4. C points from the random pattern in Fig. 7: (a) elliptic lemon; (b) hyperbolic star. The ellipse axes have been included to aid the eye, and the straight lines terminating on the $\mathrm{C}$ points are those of the line classification. The dotted grey lines are contour lines of the surface $\operatorname{det}\left(\mathscr{M}(\mathbf{R})-\mathscr{M}\left(\mathbf{R}_{0}\right)\right)$, used in the contour classification, showing that the lemon is elliptic and the star is hyperbolic. The lemon is a close up of the box (i) in Fig. 7 , the star of the box (ii). 


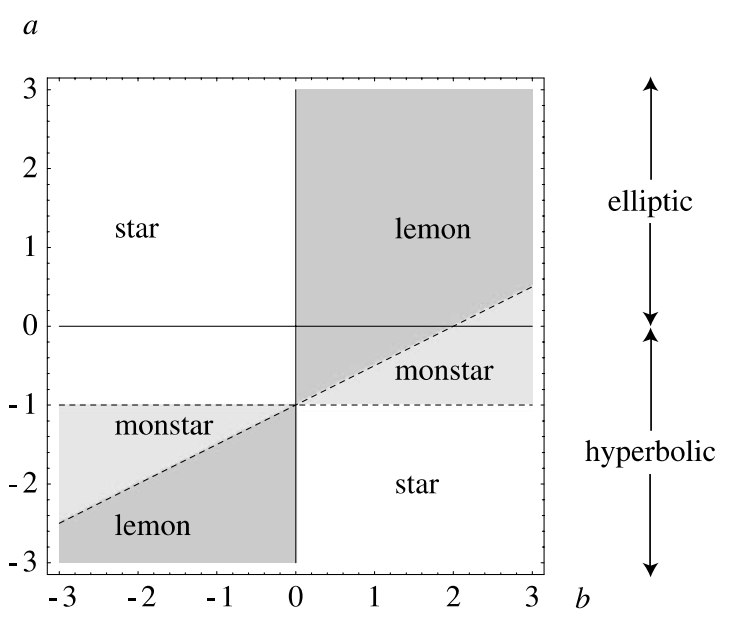

Fig. 5. Depiction in $(b, a)$ plane of the different morphological types of $\mathrm{C}$ point in the example (31). Values of $(a, b)$ giving lemons are dark grey, monstars are light grey, and stars are white. Elliptic points have $a>0$, hyperbolic $a<0$.

unchanged), so all 12 types of $\mathrm{C}$ point are realizable from this example. Both $a$ and $b$ are required to realize all of the types, so the Venn diagram of different morphological types, shown in Fig. 5, is two-dimensional, unlike the umbilic point case where it is only one-dimensional [25].

The number of $\mathrm{C}$ points in a given area $A$ of the plane, $\#_{\mathrm{C}, A}$ is

$\#_{\mathrm{C}, A}=\int_{A} \mathrm{~d}^{2} \mathbf{R} \delta\left(S_{1}\right) \delta\left(S_{2}\right)\left|D_{\mathrm{I}}\right|$

The $\delta$-functions pick out the $\mathrm{C}$ points only, and the modulus of $D_{\text {I }}$ gives the correct jacobian for an integral in real space. It is analogous to the expressions given in $[19,20]$ for phase singularity densities; this is the equivalent form for phase singularities in $\sigma$. Eq. (35) gives the same result if $S_{1}, S_{2}$ are replaced by $T_{1}, T_{2}$. The density of lemon, monstar, star, elliptic, hyperbolic, LH, RH types (or a mixture) in $A$ can be calculated by multiplying the integrand in Eq. (35) by the appropriate product of step functions $\Theta\left(D_{\mathrm{L}}\right), \Theta\left(D_{\mathrm{I}}\right), \Theta\left(S_{3}\right)$, etc.

If the polarization field is nonparaxial, the polarization is circular along lines, called $\mathrm{C}^{\mathrm{T}}$ lines (or $\mathrm{C}$ lines) [4,7], which cross an (arbitrary) plane at points. The scalar $\varphi=\mathbf{E} \cdot \mathbf{E}$, which is defined when the field is nonparaxial, has phase singularities along $\mathrm{C}^{\mathrm{T}}$ lines. The scalar $\sigma$ is not defined in these fields, because the Stokes parameters are not welldefined.

\section{4. $L$ lines and relative singularities}

The second type of singularity we consider are places where the polarization is linear, that is, when the third Stokes parameter $S_{3}=0$. vanishes. The locus where this happens is therefore codimension 1, giving lines in the transverse plane they were introduced by Nye [2], who called them s lines. Following more recent work $[8,16]$, we call them $\mathrm{L}$ lines. On $\mathrm{L}$ lines, the handedness of the ellipse is not defined (singular), consistent with the fact that the singularities of fields specified by a discrete parameter (i.e., sign $S_{3}$ ) have codimension 1 ; in this case, the $\mathrm{L}$ lines separate regions of $\mathrm{RH}$ and $\mathrm{LH}$ polarization.

The vanishing of $S_{3}=T_{3}$ can be interpreted by Eq. (13), as the vanishing of the cross product of $\mathbf{p}$ and $\mathbf{q}$, that is, the vectors are (anti)parallel, and the complex vector $\mathbf{E}$ becomes a real vector times a complex phase factor. Both the plane angle of this real vector (equivalent to $\gamma_{0}$ ) and its complex phase (equivalent to $\chi_{0}$ ), change continuously along the L line. In terms of circular components, Eq. (13) implies that $\left|\psi_{\mathrm{R}}\right|=\left|\psi_{\mathrm{L}}\right|$ on an L line, and the phases $\chi_{R}, \chi_{L}$ change continuously along it. Unlike $\mathrm{C}$ points, which have a rich topological structure, there is no characteristic structure around an $\mathrm{L}$ line; generically, $S_{3}$ passes through zero smoothly, and the angles $\gamma_{0}, \chi_{0}$ change smoothly along the singular line. The analog of $\mathrm{L}$ lines for surfaces is where the gaussian curvature, defined as the hessian determinant $H \equiv \operatorname{det} \mathscr{H}=0$. These are the parabolic lines [26], separating regions of positive curvature from negative curvature. Their significance in focusing was described by Berry [43], who showed that caustic lines in the far field are deformed images of the parabolic lines on the focusing surface.

The vector $\mathbf{U}_{\mathrm{L}}$, defined to point in the direction of an L line with the $\mathrm{RH}$ region on the left, is easily defined: since $\nabla S_{3}$ points into the $\mathrm{RH}$ region, it is

$\mathbf{U}_{\mathrm{L}}=\left(S_{3 y},-S_{3 x}\right)$. 
Note that, with this definition, $\left|\mathbf{U}_{\mathrm{L}}\right|=\left|\nabla S_{3}\right|$. The length of $\mathrm{L}$ line in an area $A$ of the plane is given by the integral

$\ell_{\mathrm{L}, A}=\int_{A} \mathrm{~d}^{2} \mathbf{R} \delta\left(S_{3}\right)\left|\nabla S_{3}\right|$,

and the number of $\mathrm{L}$ line crossings on a specified line $L$ in the plane is given by

$\#_{\mathrm{L}, L}=\int_{L} \mathrm{~d} R \delta\left(S_{3}\right)\left|S_{3}^{\prime}\right|$,

where the integral is along $L$, and $S_{3}^{\prime}$ is the derivative of $S_{3}$ along this line.

There are two further types of singularity, found on L lines, which we call relative singularities, because their singular nature is relative to a chosen phase or orientation. The first are the zeros of the instantaneous real field $\mathbf{E}_{\alpha}^{\mathrm{Re}}$; these are generically points, which may have a source, sink, circulation, spiral or saddle morphology, and are called wave disclinations [2]. They can only exist on L lines, because otherwise $E_{\gamma}^{\mathrm{Re}}$ is never zero. Throughout an entire cycle, $0 \leqslant \chi \leqslant 2 \pi$, each point on the $\mathrm{L}$ line is a disclination twice, and the disclination points move along the $\mathrm{L}$ line as $\chi$ increases. Disclinations are relative singularities, since their position is phase-dependent, unlike C points and $\mathrm{L}$ lines, whose position is independent of phase. The disclination index is defined to be the Poincaré index of the zero of the real field $\mathbf{E}_{\gamma}^{\mathrm{Re}}$, which may be shown to be the sign of the function $D_{\text {disc }}$, (cf. Eqs. (1), (2) and (20))

$D_{\text {disc }}=E_{\chi x, x}^{\mathrm{Re}} E_{\chi y, y}^{\mathrm{Re}}-E_{\chi x, y}^{\mathrm{Re}} E_{\chi y, x}^{\mathrm{Re}}$.

On an $\mathrm{L}$ line, the minor semiaxis $\mathbf{q}_{0}$ is zero, and $\mathbf{E}=\mathbf{p}_{0} \exp \left(\mathrm{i} \chi_{0}\right)$ from (7), implying that $\mathbf{E}_{\alpha}^{\mathrm{Re}}=$ $\mathbf{p}_{0} \cos \left(\chi_{0}-\chi\right)$, which is zero (a disclination) when $\chi_{0}-\chi=\pi / 2 \bmod \pi$. Disclinations for different $\chi$ can therefore be identified by the crossings of the phase contours of $\varphi$ with $\mathrm{L}$ lines. On the phase sphere, they correspond to particular points (given by the phase $\chi$ ) on the equator.

The analog of disclinations on the Poincaré sphere are component zeros or component vortices [13-15]: if a point of linear polarization on an L line has orientation angle $\gamma_{0}$, the component of $\mathbf{E}$ in the direction $\gamma_{0}+\pi / 2$ is zero (a phase singularity). These singularities, relative to a choice of $\gamma_{0}$, are readily measurable using polarizers. Hajnal [5] section 6 derived a topological result, relating the number of disclinations on a closed L loop with the total index of $\mathrm{C}$ points enclosed, namely that the sum of disclination indices on the $\mathrm{L}$ line is equal to $\pm 2 \pi$ times the $C$ point index enclosed by the L loop. The analogous statement for component zeros was made by $[14,15]$. In the following, we prove this result more simply than in [15].

Consider a place on an L line (not necessarily closed) where $E_{y}=0$; at this point, $S_{1}=S_{0}$, $S_{2}=S_{3}=0$ and through the point, $\gamma_{0}$ is smoothly changing. We will show that the sense of rotation of $\gamma_{0}$, along the $\mathrm{L}$ line in the direction of $\mathbf{U}_{\mathrm{s}}$, is equal to the dislocation strength (2) of the component zero. The rate of change of $\gamma_{0}$ along the $\mathrm{L}$ line is

$$
\begin{aligned}
\frac{\mathbf{U}_{\mathrm{s}}}{\left|\mathbf{U}_{\mathrm{s}}\right|} \cdot \nabla \gamma_{0} & =\frac{\left(S_{3 y}, S_{3 x}\right)}{\left|\mathbf{U}_{\mathrm{s}}\right|} \cdot \frac{\left(S_{1} S_{2 x}-S_{2} S_{1 x}, S_{1} S_{2 y}-S_{2} S_{1 y}\right)}{S_{1}^{2}+S_{2}^{2}} \\
& =\frac{1}{S_{1}\left|\mathbf{U}_{\mathrm{s}}\right|}\left(S_{2 x} S_{3 y}-S_{2 y} S_{3 x}\right) \\
& \propto \operatorname{sign} S_{1} \operatorname{sign}\left(S_{2}+\mathrm{i} S_{3}\right) \\
& \propto p_{y, x} q_{y, y}-p_{y, y} q_{y, x},
\end{aligned}
$$

where Eqs. (10) and (36) have been used in the first line, $S_{2}=0$ at the zero point in the second, and $S_{1}>0\left(E_{y}=0\right)$ in the third. The result in the third equality is the sign of the phase singularity in $E_{y}$, and this result generalizes for a zero in any component by rotating the plane. Note that the second line involves the sign of the topological charge of the Stokes field $S_{2}+\mathrm{i} S_{3}$ [9]. Around a closed L loop enclosing a $\mathrm{RH}$ region, the $\mathrm{C}$ point index enclosed, by Eqs. (20) and (40) equals the total topological charge of the component zeros on the $\mathrm{L}$ line, for any choice of $\gamma_{0}$. If a LH region is enclosed, the sign of $\mathbf{U}_{\mathrm{s}}$ must be reversed, so the $\mathrm{C}$ point index enclosed is minus the sum of topological charges of the component zeros. Similar results for disclinations hold, but $\chi_{0}$ replaces $\gamma_{0}$ (and other quantities in the derivation are replaced from their Poincaré sphere to their phase sphere versions). An ellipse pattern around a closed L loop containing a $\mathrm{C}$ point is shown in Fig. 6 .

The corresponding singularities in nonparaxial fields, where the polarization is linear, are lines rather than surfaces (they have codimension 2, not 


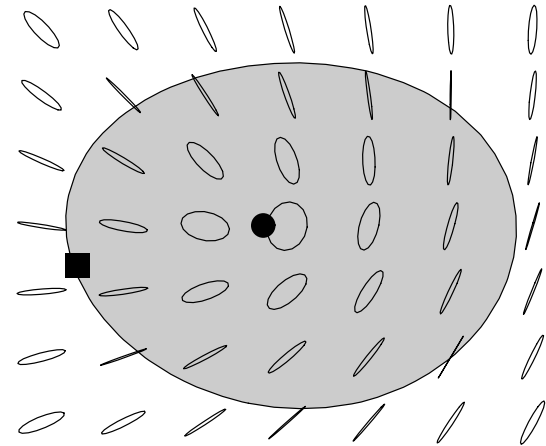

Fig. 6. The ellipse field near a closed L loop, enclosing a region of $\mathrm{RH}$ polarization (shaded). One $\mathrm{C}$ point, marked $\mathbf{O}$, is in the region, and there is one component zero $E_{y}=0$ on the enclosing $\mathrm{L}$ line, marked $\mathbf{\square}$. The whole picture is the box marked (i) in Fig. 7, and the field near the C point is depicted in Fig. 4(a).

1 as in the paraxial case). They are called $\mathrm{L}^{\mathrm{T}}$ lines (or L lines) [4,7], and are singularities of the direction of the normal to the polarization ellipse. The difference in codimension reflects the topological difference between the Poincaré sphere and the Majorana sphere $[11,40]$.

\section{Polarization singularities in isotropic random fields}

We now apply our study to polarization singularities 'in the wild', that is, in isotropic gaussian random complex vector fields. Since we are assuming monochromaticity, for each realization of the random function, there is a well-defined ellipse at each point in the plane with a well-defined intensity, eccentricity and (possibly singular) orientation angle and rectifying phase. The scalar analogs of these fields have been studied as a model for speckle fields [44,45], and the scalar singularity behaviour by $[11,19-22,24]$. Other studies have calculated the statistics of geometric properties of the polarization ellipse $[46,47]$ and Stokes parameter statistics [48-50], but not necessarily for fields (instead, the randomness is in time, which is not relevant here); by ergodicity of the gaussian model, spatial averages are equal to ensemble averages.

The fields are taken to be the sum of plane waves with random phases, equally distributed in direction, and are described in Appendix A; the circular components $\psi_{\mathrm{R}}, \psi_{\mathrm{L}}$, and equivalently the cartesian components $E_{x}, E_{y}$, are independent identically distributed circular gaussian fields, whose derivatives are also independent gaussian random fields. The two-dimensional model is a generalization of that considered in [19], where more details may be found. The random field, as defined, is extremely democratic; for instance, the areas of $\mathrm{LH}$ and $\mathrm{RH}$ polarization are equal on average, and only generic singularities contribute to their averages. The details of the calculations may be found in Appendix B, and only the results are discussed here. The random surface we will compare with is simply a real scalar component of this complex gaussian random field model; it is discussed in more detail in [25,27-29]. The pattern in one square wavelength of a random wave with the ring spectrum is shown in Fig. 7.

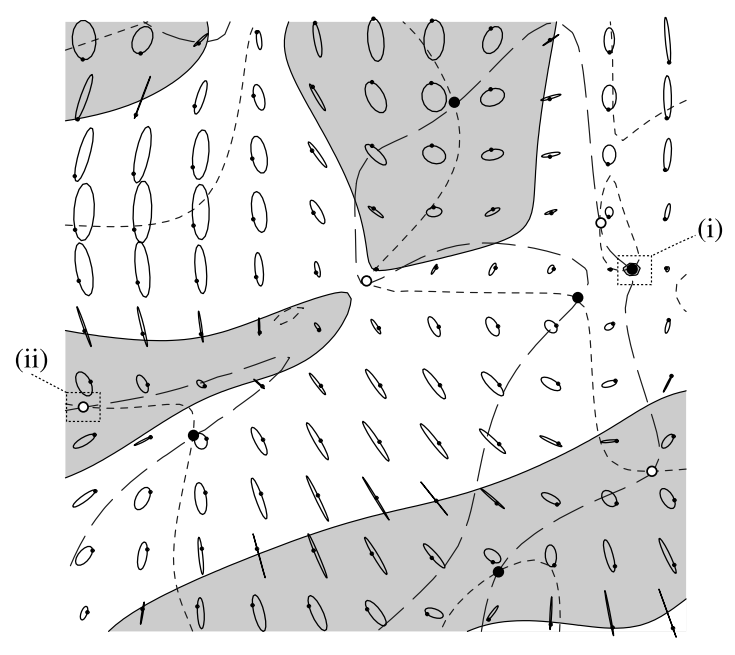

Fig. 7. One square wavelength $\left(\left(2 \pi / K_{\mathrm{d}}\right)^{2}\right)$ of a random paraxial field with the ring spectrum, constructed by superposing 50 monochromatic random waves from Eq. (A.1). The RH regions are shaded, and separated from the LH regions by L lines (solid lines). $\mathrm{C}$ points are represented by when the index is $+1 / 2$, $\mathrm{O}$ when $-1 / 2$, and all $+1 / 2 \mathrm{C}$ points are lemons here. The position of the real vector $p$ is mared by a dot on each ellipse, and a lines by dashed curves: $\operatorname{Re} \sigma=0$ (long dashes), $\operatorname{Im} \sigma=0$ by short dashes. A close up of the field in the box marked (i) is shown in Fig. 6 (a further close up in Fig. 4(a)) and the box marked (ii) is shown in Fig. 4(b). 
The density of C points in the paraxial plane $d_{\mathrm{C}}$ is the ensemble average of the density expression (35), and is

$d_{\mathrm{C}}=\frac{K_{2}}{2 \pi}=0.15915 K_{2}$,

where $K_{2}$ denotes the second moment of the power spectrum [19]. This result agrees with, and can simply be derived from, the fact that $\mathrm{C}$ points are dislocations in the circular components $\psi_{\mathrm{L}}, \psi_{\mathrm{R}}$; the density of dislocations $d_{\mathrm{D}}$ in a random complex scalar paraxial wavefield was calculated in [19-21] to be

$d_{\mathrm{D}}=\frac{K_{2}}{4 \pi}$.

The total $\mathrm{C}$ point density is the sum of dislocations in $\psi_{\mathrm{R}}, \psi_{\mathrm{L}}$, each of which contributes a density of $d_{\mathrm{D}}$, so $d_{\mathrm{C}}=2 d_{\mathrm{D}}$, and the densities of $\mathrm{LH}$ and $\mathrm{RH}$ $\mathrm{C}$ points are equal. The mean $\mathrm{C}$ point index is also zero - a consequence of the global neutrality of dislocation strength, discussed in [21,23]. In Fig. 7, there are $9 \mathrm{C}$ points: five lemons (two $\mathrm{RH}$, three LH) and four stars (two RH, two LH). The expected number, in one square wavelength of the ring spectrum $\left(4 \pi^{2} / K_{\mathrm{d}}^{2}\right)$, is $2 \pi$, slightly fewer than in this sample. The total topological charge in this square is 1 . The interpretation of $\mathrm{C}$ points as dislocations means that many statistical results previously derived for dislocations apply to $\mathrm{C}$ points; for instance, [20] computed the dislocation densities in anisotropic fields and in a longitudinal plane, in addition to the paraxial plane; these results apply directly to $\mathrm{RH}$ and $\mathrm{LH} \mathrm{C}$ points.

In any realization of the random field (i.e., a sample function), the densities of $\mathrm{C}$ singularities at two separated points are not independent, but depends on their separation. In our isotropic model, the direction of separation is unimportant, and the average of two local $\mathrm{C}$ point densities (of possibly different types), at points separated by $R=|\mathbf{R}|$, is given by the correlation functions, normalized by the $\mathrm{C}$ point density. This implies that when the densities are independent (for instance, for large $R$ ), the correlation is 1 . The theory of correlations for wave dislocations in the plane is studied by $[19,21,23,51]$, using results and notations from the statistical mechanics of fluids and plasmas [52]; the dislocation correlation functions are themselves functions of the field autocorrelation function $C(R)$, defined in Eq. (A.7). We will use the notation of [19], to which the reader is referred for details; for instance, the partial correlation function between types $i, j$ is denoted by $g_{i j}(R)=g_{i j}$, which equals $g_{j i}$ by isotropy.

Correlations are examined between four different types of $\mathrm{C}$ point-index: $+1 / 2 \mathrm{RH},-1 / 2 \mathrm{RH}$, $+1 / 2 \mathrm{LH},-1 / 2 \mathrm{LH}$. Of these, all RH densities are independent of LH densities (since they are dislocations of the independent fields $\psi_{\mathrm{R}}, \psi_{\mathrm{L}}$ ), so, in an obvious notation

$$
\begin{aligned}
g_{+\mathrm{R}+\mathrm{L}} & =g_{+\mathrm{R}-\mathrm{L}}=g_{-\mathrm{R}+\mathrm{L}}=g_{-\mathrm{R}-\mathrm{L}}=g_{+\mathrm{L}+\mathrm{R}} \\
& =g_{+\mathrm{L}-\mathrm{R}}=g_{-\mathrm{L}+\mathrm{R}}=g_{-\mathrm{L}-\mathrm{R}}=1 .
\end{aligned}
$$

The partial correlations between $\mathrm{C}$ points of the same handedness is clearly the same as the corresponding correlations for dislocations, denoted by $g_{++}=g_{--}, g_{+-}=g_{-+}$, so

$g_{+\mathrm{R}+\mathrm{R}}=g_{-\mathrm{R}-\mathrm{R}}=g_{+\mathrm{L}+\mathrm{L}}=g_{-\mathrm{L}-\mathrm{L}}=g_{++}$,

$g_{+\mathrm{R}-\mathrm{R}}=g_{+\mathrm{L}-\mathrm{L}}=g_{-\mathrm{R}+\mathrm{R}}=g_{-\mathrm{L}+\mathrm{L}}=g_{+-}$.

The total number correlation function $g=g(R)$ is the sum of all the 16 partial correlations, divided by 16 in order that $g(R) \rightarrow 1$ as $R \rightarrow \infty$,

$$
\begin{aligned}
g & =\frac{1}{16} \sum_{i j} g_{i j}=\frac{1}{2}+\frac{1}{4}\left(g_{++}+g_{+-}\right) \\
& =\frac{1}{2}+\frac{g_{\mathrm{D}}}{2},
\end{aligned}
$$

where $i, j=+\mathrm{R},+\mathrm{L}$, etc. and $g_{\mathrm{D}}$ represents the dislocation number correlation function [19].

It is also possible to calculate the $C$ point charge correlation function $g_{\mathrm{I}}$, that is the total correlation function with local densities weighted by their index (for dislocations, by topological charge). It is

$$
\begin{aligned}
g_{\text {I }} & =\frac{1}{16} \sum_{i j} \operatorname{sign}(i j) g_{i j}=\frac{1}{4}\left(g_{++}-g_{+-}\right) \\
& =\frac{g_{Q}}{2},
\end{aligned}
$$

where $g_{Q}$ is the dislocation charge correlation function [19,21]. The C point correlation functions $g, g_{\mathrm{I}}, g_{++}$, and $g_{+-}$are shown in Fig. 8, for the three spectra defined in Appendix A. Therefore, just as dislocations [19,23,51], C points 'screen' 

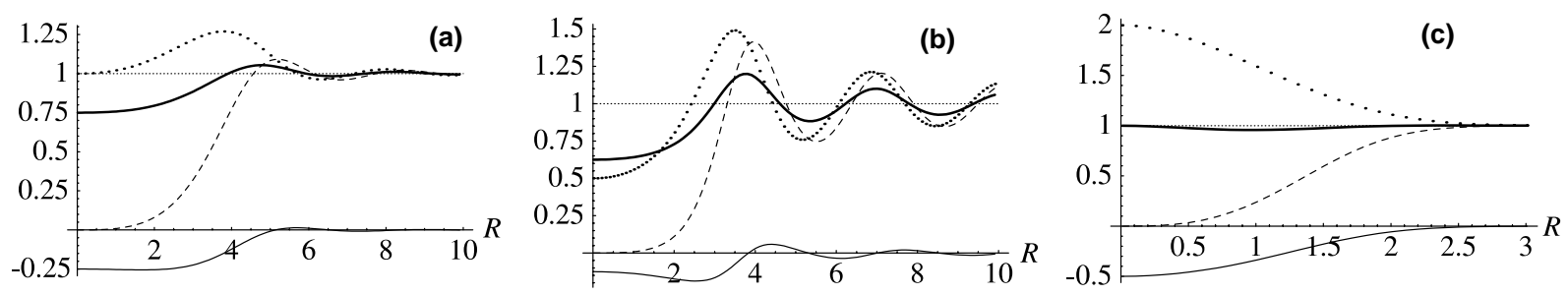

Fig. 8. C point correlation functions, $g$ (thick line), $g_{\mathrm{I}}$ (thin line), $g_{++}$(dashed line), $g_{+-}$(dotted line), for the three paraxial spectra are described in Appendix A: (a) disk spectrum; (b) ring spectrum; (c) gaussian spectrum. In (a) and (b), $R$ is plotted in units of $1 / K_{\mathrm{d}}$, and in (c), in units of $1 / K_{\sigma}$.

each other, satisfying the $\mathrm{C}$ point analog of the first Stillinger-Lovett sum rule [52]

$d_{\mathrm{C}} \int \mathrm{d}^{2} \mathbf{R} g_{\mathrm{I}}(R)=2 \pi d_{\mathrm{C}} \int_{0}^{\infty} \mathrm{d} R R g_{\mathrm{I}}(R)=-1$,

that is, each $\mathrm{C}$ point is surrounded by a cloud of $\mathrm{C}$ points of the opposite index. Note that screening only takes place between $\mathrm{C}$ points of the same handedness. These screening results are discussed further and compared with simulation data in [53].

The overall density of $\mathrm{C}$ points may be compared to the number of nonparaxial $\mathrm{C}^{\mathrm{T}}$ line crossings of a plane, which were calculated for isotropic fields in [7], Eq. (5.10). After changing to a correct plane projected spectrum [19], the density is

$d_{\mathrm{C}^{\mathrm{T}}}=\frac{3 K_{2}}{4}\left(\frac{3}{10 \pi}+\frac{1}{5 \sqrt{3}}\right)=0.15822 K_{2}$.

The numerical value is therefore very close to $d_{\mathrm{C}}$, although no approximation has been made in either calculation and the analytic forms in (41) and (49) are rather different. Physically, there is a significant difference in the two fields - the circular polarization ellipses of the $\mathrm{C}^{\mathrm{T}}$ points do not lie in the plane in which the density is measured. This phenomenon, where two random quantities which are similar physically and give numerically close but analytically different values, also appears for the density of nonparaxial $\mathrm{L}^{\mathrm{T}}$ lines crossing a plane, given in [7], which is close numerically to $d_{\mathrm{C}^{\mathrm{T}}}$. It appears again in the comparison of densities of $\mathrm{L}$ lines and parabolic lines, described below.

The $\mathrm{C}$ point density may also be compared to the umbilic point density on isotropic random surfaces; the total umbilic point density $d_{\mathrm{U}}$ was calculated in [25] to be
$d_{\mathrm{U}}=\frac{K_{6}}{4 \pi K_{4}}$.

The factor $K_{6} / K_{4}$ involves higher moments than the $\mathrm{C}$ point density, because umbilic points are singularities in higher derivatives of the field. Apart from this spectral factor, the density is half that of $\mathrm{C}$ points, or equal to the density of RH C points.

The fractional densities $\alpha_{C}$ of the lemon, monstar, star fractions of $\mathrm{C}$ points are calculated in Appendix B (for total density, multiply by $d_{\mathrm{C}}$ )

$$
\begin{aligned}
& \alpha_{\mathrm{C}, \mathrm{star}}=0.5, \\
& \alpha_{\mathrm{C}, \mathrm{monstar}}=0.05279, \\
& \alpha_{\mathrm{C}, \text { lemon }}=0.44721 .
\end{aligned}
$$

These are precisely the same as the fractions for umbilic points, calculated in [25] Eq. (41). Since the star singularities are precisely those with index $-1 / 2, \alpha_{\mathrm{C}, \mathrm{star}}$ must be $1 / 2$ since the total charge density is zero. Just over a 10 th of the index $+1 / 2$ points are monstars, and the rest are lemons. The fractions are the same for $\mathrm{RH}$ and $\mathrm{LH} \mathrm{C}$ points.

The density fractions for $\mathrm{C}$ points under the contour classification (E for elliptic, $\mathrm{H}$ for hyperbolic) are also calculated in Appendix B. The results for all possible combinations may be found from the following results (and (51)):

$$
\begin{aligned}
& \alpha_{\mathrm{C}, \mathrm{E}}=1 / 2, \\
& \alpha_{\mathrm{C},+\mathrm{E}}=\alpha_{\mathrm{C},-\mathrm{E}}=1 / 4, \\
& \alpha_{\mathrm{C}, \mathrm{E} \text { lemon }}=0.2348>\alpha_{\mathrm{C}, \mathrm{lemon}} / 2 .
\end{aligned}
$$

Half of the $\mathrm{C}$ points are elliptic, half are hyperbolic, and these are equally distributed in positive and negative index. However, more than half of the lemon type are elliptic, so fewer than half of the monstars can be elliptic (in order that half 
of the positive index (lemons + monstars) $\mathrm{C}$ points are elliptic). A two-dimensional Venn diagram, with areas corresponding to the six different $\alpha$ fractions, is shown in Fig. 9. This diagram should be compared with the umbilic case, in [25] Fig. 2, which is one-dimensional since for umbilic points, only stars can be elliptic (and $\alpha_{\mathrm{U}, \mathrm{E} \text { star }}=0.268$ ). Statistically, therefore, the line classification does not distinguish between $\mathrm{C}$ points and umbilic points, but the contour classification does. The sign of the morphological parameter $D_{\mathrm{L}}$, defined in Eq. (29), determines the line classification, and only involves the Stokes parameters $S_{1}$ and $S_{2}$, whereas $D_{\mathrm{C}}$, in Eq. (30), involves $S_{0}$ as well. This similarity in the line classification but difference in the contour classification was observed by Nye [3], pp. 90-91, for C point/umbilic point creation/ annihilation. It has already been stated that out of the nine $\mathrm{C}$ points in Fig. 7, five are lemons and four are stars (no monstars). Although not shown, four are hyperbolic (two lemons and two stars), and five are elliptic (three lemons and two stars).

The density $d_{\mathrm{r}}$ of any type of relative singularity (either the component zeros in a specified direction, or the disclinations for a specified phase) is

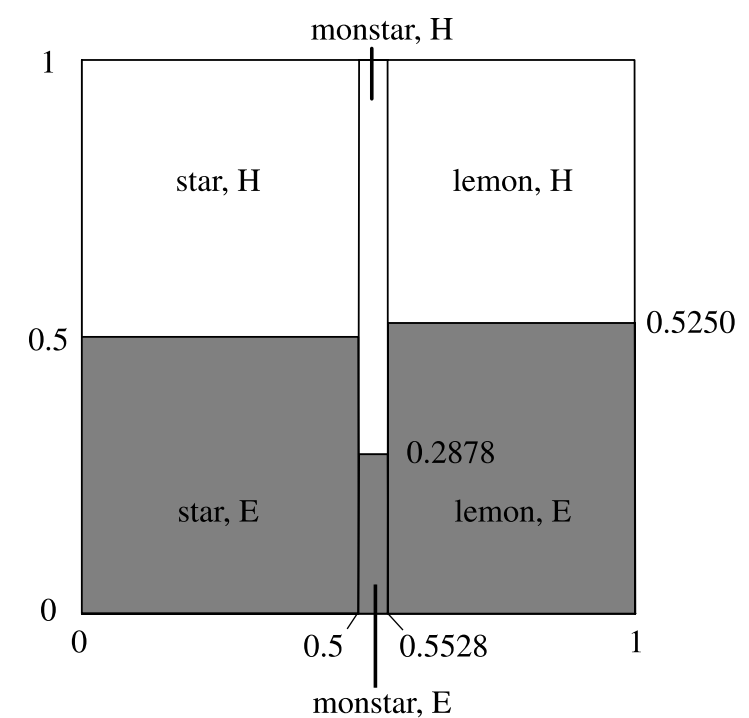

Fig. 9. Venn diagram showing the fractions of different morphological types of C points, from Eqs. (51) and (52). The areas of the sets are proportional to the fraction of that type. clearly equal to the corresponding scalar dislocation density; the zeros of a linearly polarized component are just dislocations in that scalar field; at a disclination the $x$ and $y$ components of a real vector must vanish, and these pairs of fields are all identically and independently distributed (just like the real and imaginary parts of a complex scalar field). The calculation reduces to that of dislocations in each case, so

$d_{\mathrm{r}}=d_{\mathrm{D}}=\frac{K_{2}}{4 \pi}$.

In fact, this argument may easily be extended to show that the density of any polarization state, specified either on the Poincaré or phase sphere, has the dislocation density (53).

The density $d_{\mathrm{L}}$ of L lines is shown in Appendix B to be

$d_{\mathrm{L}}=\frac{\pi}{4} \sqrt{\frac{K_{2}}{2}}=0.55536 \sqrt{K_{2}}$

and the related density of $\mathrm{L}$ lines crossing a straight line is $\sqrt{K_{2}} / 2 \sqrt{2}$. They differ by a factor of $2 / \pi$, which is general and given by the Buffon needle relation - the density of crossings of a random curve with a straight line is generally $2 / \pi$ times the length of that curve [54]. L lines may be compared statistically and morphologically to two other line morphologies on real random surfaces: zero contour lines, whose density is denoted $d_{\mathrm{z}}$, and parabolic lines (where $H=0$ ), whose density is $d_{\mathrm{p}}$. Fig. 10 shows a sample of the three types of line morphology.

The density of zero lines was computed by Longuet-Higgins [28] to be

$d_{\mathrm{z}}=\frac{K_{2}}{2 \sqrt{2}}$

and the corresponding line crossing density is $\sqrt{K_{2}} / \pi \sqrt{2}$, also shown in [28], and also following from Buffon's relation. This density is smaller than $d_{\mathrm{L}}$ by a factor of $\pi / 2$. The density of parabolic lines of an isotropic gaussian random surface can be shown to be [55]

$d_{\mathrm{p}}=\frac{2 \sqrt{5} E(4 / 5)}{3 \pi} \sqrt{\frac{K_{6}}{K_{4}}}=0.55920 \sqrt{\frac{K_{6}}{K_{4}}}$, 
where $E$ denotes the complete elliptic integral of the second kind [56], and the spectral factor $\sqrt{K_{6} / K_{4}}$ is the square root of that for umbilic points (50). Numerically, this is very close to the $\mathrm{L}$ line density, although they are not equal, as discussed above for $\mathrm{C}$ points and $\mathrm{C}^{\mathrm{T}}$ point crossings. Mathematically, the difference comes about since $S_{3}=p_{x} q_{y}-q_{x} p_{y}=0$ on an $\mathrm{L}$ line, where the second term is the product of independent random variables, whereas on a parabolic line, $H=f_{x x} f_{y y}-f_{x y}^{2}=0$, where the second term is the square of a random variable.

This difference might appear to be small as far as the $\mathrm{L}$ and parabolic densities are concerned, but leads to rather strong differences in the forms of the two sets of line patterns, and the ensuing morphologies of regions of $\mathrm{RH}$ and $\mathrm{LH}$ in polarization fields, and positive and negative gaussian curvature on random surfaces. Morphologically, L lines are closer to zero contour lines than parabolic lines, as may be seen in Fig. 10. This may be seen more clearly in the probability distribution of the values of $S_{3}, f$ and $H$ - the L lines, zero contour lines and parabolic lines are the zeros of these functions. The distribution of $S_{3}$ is symmetric about 0 from (A.16), so the $\mathrm{L}$ lines partition the plane into equal areas of $\mathrm{LH}$ and $\mathrm{RH}$ polarization, just as the zero lines of the gaussian distributed $f$ partition the plane into equal areas of $f>0$ and $f<0$. However, Longuet-Higgins [29] derived the probability distribution of the gaussian curvature (Eq. (7.14)), which is asymmetric, and in the present notation is given by (a)

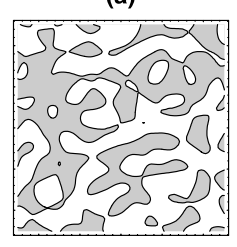

(b)

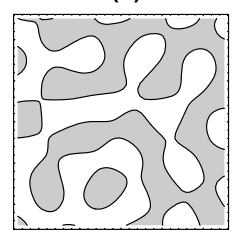

(c)

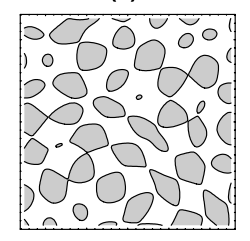

Fig. 10. Comparison of the morphology of three types of line in random waves, in 16 square wavelengths: (a) L lines; (b) zero contours of $f$; (c) parabolic lines of $f$. (a) uses the same random complex vector field as Fig. 7, and (b), (c) are the zero contours and parabolic lines over the same area of a random function $f$, constructed by superposing 50 random waves with the ring spectrum.

$$
P(H)=\frac{4}{K_{4} \sqrt{3}} \exp \left(\frac{4 H}{K_{4}}\right)\left(1-\Theta(H) \operatorname{erf}\left(\sqrt{\frac{6 H}{K_{4}}}\right)\right) .
$$

Although the mean curvature $\langle H\rangle=0$, the fraction of the plane where $H$ is positive is less than $1 /$ 2 ,

$\langle\Theta(H)\rangle=1-\frac{1}{\sqrt{3}}=0.42265<\frac{1}{2}$.

The symmetry of $p\left(S_{3}\right)$ and $p(f)$ imply that the regions of $\mathrm{RH}$ and $\mathrm{LH}$ polarizations, and positive and negative regions of $f$, in a random field, are at the percolation threshold [57-59], and there is an infinite, 'percolating' L line or zero contour (this does not mean that there cannot be finite loops as well, as in the figures). This contrasts with the asymmetric distribution of $H$, where generically there are isolated islands of positive curvature, surrounding each extremum (either maximum or minimum), surrounded by a sea of negative curvature, which is evident from Fig. 10(c). Each connected region of positive curvature contains exactly one extremum, and the distribution of extrema is statistically stationary and isotropic [28].

\section{Acknowledgements}

I am grateful to M.V. Berry, J.H. Hannay and J.F. Nye for invaluable discussions, and to I. Freund and M.S. Soskin for useful correspondence. This work was supported by the Leverhulme Trust.

\section{Appendix A. Isotropic gaussian random polariza- tion fields}

The gaussian random polarization fields used to derive the results in Section 5 are very simple each of the complex cartesian components $E_{x}, E_{y}$ is a circular gaussian random function, of the type normally considered in speckle patterns [44,45], and in the properties of wave dislocations in scalar fields [19], on which the present discussion is based. Each scalar component is a sum of plane 
wave components (neglecting the monochromatic time dependence and paraxial $z$-dependence)

$E_{\mu}=\sum_{\mathbf{K}} a_{\mathbf{K}} \exp \left(\mathrm{i}\left(\mathbf{K} \cdot \mathbf{R}+\phi_{\mu, \mathbf{K}}\right)\right), \quad \mu=x, y$,

where the $\mathbf{K}$ are transverse wavenumbers, isotropic in direction in the $x, y$ plane, the $\phi_{\mu, \mathbf{K}}$ are uniformly random phases (labelled by $\mu$ and $\mathbf{K}$ ) and $a_{\mathbf{K}}$ are spectral factors depending only on the length $K=\mathbf{K}$, ensuring that the fields (A.1) are isotropic. The sets of phases $\left\{\phi_{x, \mathbf{K}}\right\},\left\{\phi_{y, \mathbf{K}}\right\}$ are independent so $E_{x}, E_{y}$ are completely independent. All of the polarization is pure in this model since the fields are monochromatic, and all randomness is spatial. The fields (A.1) are statistically stationary and ergodic, so all spatial averages over $\mathbf{R}$ can be replaced by ensemble averages (over the $\phi_{\mathbf{K}}$ ), denoted by $\langle\bullet\rangle$; therefore

$\left\langle E_{x} E_{y}\right\rangle=0$.

From (6), the field may be rewritten in terms of circular components $\psi_{\mathrm{R}}, \psi_{\mathrm{L}}$, and, from (A.2),

$\left\langle\psi_{\mathrm{R}} \psi_{\mathrm{L}}\right\rangle=0$.

It may be shown that the above definition of the random $\mathbf{E}$ field is equivalent to

$\mathbf{E}=\sum_{\mathbf{K}} a_{\mathbf{K}} \mathbf{d}_{\mathbf{K}} \exp \left(\mathrm{i}\left(\mathbf{K} \cdot \mathbf{R}+\phi_{\mathbf{K}}\right)\right)$,

where $a_{\mathbf{K}}$ and $\phi_{\mathbf{K}}$ are as above, and $\mathbf{d}_{\mathbf{K}}$ is a random complex polarization vector for each $\mathbf{K}$, representing a point chosen at random on the Poincaré sphere,

$$
\begin{aligned}
\mathbf{d}_{\mathbf{K}}= & 4\left(\cos \left(\alpha_{\mathbf{K}} / 2\right) \exp \left(-\mathrm{i} \beta_{\mathbf{K}} / 2\right),\right. \\
& \left.\sin \left(\alpha_{\mathbf{K}} / 2\right) \exp \left(\mathrm{i} \beta_{\mathbf{K}} / 2\right)\right),
\end{aligned}
$$

where $\alpha_{\mathbf{K}}$ and $\beta_{\mathbf{K}}$ are the angles on the Poincaré sphere, as defined in the text below Eq. (15), and the factor 4 is for normalization.

Denoting any one of identically distributed and independent real gaussian random fields $p_{x}, q_{x}, p_{y}$, $q_{y}, \operatorname{Re} \psi_{\mathrm{R}}, \operatorname{Im} \psi_{\mathrm{R}}, \operatorname{Re} \psi_{\mathrm{L}}$ and $\operatorname{Im} \psi_{\mathrm{L}}$ by $f:$

$\left\langle f^{2}\right\rangle=\frac{1}{2} \sum_{\mathbf{K}} a_{\mathbf{K}}^{2}=\int \mathrm{d}^{2} \mathbf{K} \frac{\Pi(K)}{2 \pi K}$,

where the second equality follows assuming that the $\mathbf{K}$ are sufficiently finely spaced that the sum may be replaced by an integral, and $\Pi(K)$ is the radial power spectrum. The two-dimensional Fourier transform of the power spectrum, by the Wiener-Khintchine theorem, is the autocorrelation function $C(R)$, also defined by

$C(R)=\langle f(0) f(R)\rangle$.

Note that the cross correlations corresponding to the averages Eqs. (A.2), (A.3) are always 0.

The $n$th moment of $K$ with respect to the radial power spectrum $\Pi(K)$ is denoted $K_{n}$; without loss of generality $\Pi(K)$ is normalized, so $K_{0}=1 . p_{x}, p_{y}$, $q_{x}, q_{y}$, and their first derivatives are all independent (this also applies to $\xi_{\mathrm{R}}, \eta_{\mathrm{R}}, \xi_{\mathrm{L}}$ and $\eta_{\mathrm{L}}$ ) and again denoting any of the fields by $f$ :

$\left\langle f^{2}\right\rangle=K_{0}=1$,

$\left\langle f_{x}^{2}\right\rangle=\left\langle f_{y}^{2}\right\rangle=\frac{K_{2}}{2}$.

Because of this independence, the normalized probability density function of each of these fields is

$$
\begin{aligned}
P\left(f, f_{x}, f_{y}\right) & =P(f) P\left(f_{x}\right) P\left(f_{y}\right) \\
& =\frac{2}{(2 \pi)^{3 / 2} K_{2}} \exp \left(-\frac{f^{2}}{2}-\frac{2\left(f_{x}^{2}+f_{y}^{2}\right)}{K_{2}}\right) .
\end{aligned}
$$

The statistical model for random surfaces is equivalent to any one of the fields denoted by $f$ here $[28,25]$. It should be noted that $[28,25]$ use the notation $M_{n}$ for $K_{n}$.

Three particular spectra which the results apply to are the following:

- Disk spectrum. This is a polarization speckle pattern from a uniformly illuminated circular scatterer, with radius $r$ and distance $L$ from the plane; for wavelength $\lambda$, writing $K_{\mathrm{d}}=2 \pi /$ $\Lambda=2 \pi r / \lambda D$, its power spectrum is a radial step function, so

$K_{n, \text { disk }}=\frac{2 K_{\mathrm{d}}^{n}}{2+n}$

and

$$
C_{\text {disk }}(R)=\frac{2 J_{1}\left(K_{\mathrm{d}} R\right)}{K_{\mathrm{d}} R},
$$

where $J_{1}$ denotes the first order Bessel function. This spectrum is frequently considered in 
speckle theory $[45,44]$, and its phase singularities have been well-studied [22].

- Ring spectrum. As above, but the scatterer is now a ring of negligible thickness; all of the $\mathbf{K}$ vectors therefore have the same length (the power spectrum is a $\delta$-function), and

$K_{n, \text { ring }}=K_{\mathrm{d}}^{n}$

and

$C_{\text {ring }}(R)=J_{0}\left(K_{\mathrm{d}} R\right)$,

where $J_{0}$ denotes the zeroth-order Bessel function.

- Gaussian spectrum. This is the field from a gaussian scatterer, with standard deviation $K_{\sigma}$, so

$K_{n, \text { gauss }}=2^{n / 2}\left(\frac{n}{2}\right) ! K_{\sigma}^{n}$

and

$C_{\text {gauss }}(R)=\exp \left(-\frac{K_{\sigma}^{2} R^{2}}{2}\right)$.

The statistics of scalar wave singularities with these spectra were described in [11]. It is possible to show, using this model, that the probability distribution $P\left(S_{i}\right)$ of the Stokes parameters $S_{i}, i=1,2,3$, is

$P\left(S_{i}\right)=\frac{\exp \left(-\left|S_{i}\right| / 2\right)}{4}$,

agreeing with the calculations of [48-50].

\section{Appendix B. Calculations of singularity densities}

In order to calculate the statistical density of $\mathrm{C}$ points (including $\mathrm{C}$ points of certain types $(\mathrm{RH}$ or LH, star, lemon or monstar, elliptic or hyperbolic)), we use the fact that the gaussian random field $\mathbf{E}$ (A.1), (A.4) is ergodic, and replace the spatial integral in (35) with ensemble averaging, so the density $d_{\mathrm{C}}$ of $\mathrm{C}$ points is

$d_{\mathrm{C}}=\left\langle\delta\left(S_{1}\right) \delta\left(S_{2}\right)\left|D_{\mathrm{I}}\right|\right\rangle$.

It is easier to evaluate (B.1) using the phase parameters $T_{1}, T_{2}$ instead of the Stokes parameters, since the phase parameters are invariant with respect to rotations of the plane. Also, the density of any particular type of $\mathrm{C}$ point may be evaluated, with an appropriate step $\Theta$-function on the morphological parameters, $S_{3}, D_{\mathrm{I}}, D_{\mathrm{L}}, D_{\mathrm{C}}$, which is denoted for now simply by $\Theta$, so, generalizing (B.1),

$d_{\mathrm{C}, \text { type }}=\left\langle\delta\left(T_{1}\right) \delta\left(T_{2}\right)\left|T_{1 x} T_{2 y}-T_{2 x} T_{1 y}\right| \Theta\right\rangle$.

As in other derivations of singularity densities $[7,11,19]$, the calculation is simpler if an appropriate coordinate system is chosen to start with; we will represent $\mathbf{p}, \mathbf{q}$ in polar coordinates as $\left(p, \theta_{0}\right)$, and $(q$, $\left.\theta_{0}+\theta\right)$. The phase parameters $T_{1}, T_{2}$, their derivatives, and the signs of the morphological parameters are unchanged when $\theta_{0}$ is averaged in (B.2), so

$$
\begin{aligned}
d_{\mathrm{C}, \mathrm{type}}= & \frac{8}{\pi} \int_{0}^{\infty} \mathrm{d} p \int_{0}^{\infty} \mathrm{d} q \int_{0}^{2 \pi} \mathrm{d} \theta p q \delta\left(p^{2}-q^{2}\right) \\
& \times \delta(2 p q \cos \theta) \times \exp \left(-2\left(p^{2}+q^{2}\right)\right) I_{1},
\end{aligned}
$$

where $I_{1}$ represents all of the integrals involving first derivatives, including $\Theta$. The $\delta$-functions are easy to integrate (with respect to $q$ and $\theta$ ), and the integral becomes

$d_{\mathrm{C}, \mathrm{type}}=\frac{16}{\pi} \int_{0}^{\infty} \mathrm{d} p \frac{p^{2}}{4 p^{3}} \exp \left(-4 p^{2}\right) I_{1(q=p, \cos \theta=0)}$

and the notation $I_{1(q=p, \cos \theta=0)}$ is obvious. $\cos \theta$ has two zeros in the range $0 \leqslant \theta \leqslant 2 \pi$, the first $(\theta=\pi / 2)$ when $\mathbf{p}, \mathbf{q}$ are $\mathrm{RH}$, the other LH. The distributions of $\mathrm{C}$ points of any other type are the same whether the points are $\mathrm{RH}$ or $\mathrm{LH}$, and it will be assumed in the following that the type does not involve handedness.

The jacobian $\left|T_{1 x} T_{2 y}-T_{2 x} T_{1 y}\right|$ now simplifies

$$
\begin{aligned}
\left|T_{1 x} T_{2 y}-T_{2 x} T_{1 y}\right|= & 4 p^{2} \mid p_{x, x} p_{y, y}+p_{x, x} q_{x, y} \\
& -p_{y, y} q_{y, x}+q_{x, x} q_{y, y} \\
& -p_{x, y} p_{y, x}-p_{x, y} q_{x, x} \\
& -q_{x, y} q_{y, x}+p_{y, x} q_{y, y} \mid .
\end{aligned}
$$

Rewriting the first derivatives as a vector

$\mathbf{V}=2 / \sqrt{K_{2}}\left(p_{x, x}, p_{x, y}, p_{y, x}, p_{y, y}, q_{x, x}, q_{x, y}, q_{y, x}, q_{y, y}\right)$,

the jacobian in (B.5) can be written as a quadratic form, and after rescaling (to remove factors in the 
gaussian), is equal to $\left(K_{2} / 4\right)^{4}|\mathbf{V} \cdot \boldsymbol{\Xi} \cdot \mathbf{V}|$, with $\boldsymbol{\Xi}$ the $8 \times 8$ symmetric matrix with entries $0, \pm 1 / 2$, from (B.4). Therefore

$$
\begin{aligned}
d_{\mathrm{C}, \mathrm{type}}= & \frac{16}{\pi}\left(\frac{K_{2}}{4 \pi^{4}}\right) \int_{0}^{\infty} \mathrm{d} p p \exp \left(-4 p^{2}\right) \\
& \times \int \mathrm{d}^{8} \mathbf{V}|\mathbf{V} \cdot \mathbf{\Xi} \cdot \mathbf{V}| \exp \left(-V^{2}\right) .
\end{aligned}
$$

Now we perform a linear transformation $\mathbf{V} \rightarrow$ $\mathbf{W}=\boldsymbol{\Gamma} \mathbf{V}$ with $\boldsymbol{\Gamma}$ an orthogonal matrix diagonalizing $\boldsymbol{\Xi}$ :

$\boldsymbol{\Gamma}^{\mathrm{T}} \boldsymbol{\Xi} \boldsymbol{\Gamma}=\operatorname{diag}\{1,1,-1,-1,0,0,0,0\}$.

Such a $\boldsymbol{\Gamma}$ can always be found because $\boldsymbol{\Xi}$ is real symmetric. The jacobian of this transformation is 1 since $\Gamma$ is orthogonal. Defining $\alpha_{\text {type }}=2 \pi /$ $K_{2} d_{\text {C,type }}$, after the transformation, we may write

$$
\begin{aligned}
\alpha_{\text {type }}= & \frac{1}{\pi^{4}} \int \mathrm{d}^{8} \mathbf{W} \mid W_{1}^{2}+W_{2}^{2}-W_{3}^{2} \\
& -W_{4}^{2} \mid \exp \left(-W^{2}\right) \Theta_{W}
\end{aligned}
$$

where any expression in $\Theta$ has been appropriately changed to the $W_{i}$ coordinates. The total density of $\mathrm{C}$ points may therefore be confirmed by taking $\Theta_{W}=1$ always in (B.9). By transforming $W_{1}, \ldots, W_{6}$ to

$W_{1}=r \cos \theta \cos \phi_{1}$,

$W_{2}=r \cos \theta \sin \phi_{1}$,

$W_{3}=r \sin \theta \cos \phi_{2}$,

$W_{4}=r \sin \theta \sin \phi_{2}$,

$W_{5}=r u \cos \phi_{3}$,

$W_{6}=r u \sin \phi_{3}$,

$0 \leqslant r, u<\infty, \quad 0 \leqslant \theta \leqslant \pi / 2$,

$0 \leqslant \phi_{1}, \phi_{2}, \phi_{3} \leqslant 2 \pi$,

with jacobian $r^{5} u \sin (2 \theta) / 2$. With $\Theta_{W}=1$, this integral can easily be shown to be 1 , confirming that $d_{\mathrm{C}}=K_{2} / 2 / \pi$, justified in Eq. (41) a different way. Otherwise, $W_{7}, W_{8}$ may be integrated (they do not appear in any of the morphological parameters), and

$$
\begin{aligned}
\alpha_{\mathrm{type}}= & \frac{1}{2 \pi^{3}} \int_{0}^{\infty} \mathrm{d} r \int_{0}^{\infty} \mathrm{d} u \\
& \times \int_{0}^{\pi / 2} \mathrm{~d} \theta \int \mathrm{d}^{3} \phi r^{7} u \sin 2 \theta|\cos 2 \theta| \\
& \times \exp \left(-r^{2}\left(1+u^{2}\right)\right) \Theta,
\end{aligned}
$$

where the morphological parameters, in terms of $r$, $\theta, \phi_{1}, \phi_{2}, \phi_{3}, u$, are written

$D_{\text {IW }}=\cos 2 \theta$,

$D_{\mathrm{LW}}=15-28 \cos 2 \theta+11 \cos 4 \theta$

$$
-4 \cos \left(3 \phi_{1}+\phi_{2}\right) \sin 2 \theta(1+\cos 2 \theta),
$$

$D_{\mathrm{CW}}=\cos ^{2} 2 \theta-2 u^{2}\left(1-\cos \left(\phi_{1}+\phi_{2}-2 \phi_{3}\right) \sin 2 \theta\right)$,

where overall positive multipliers have been ignored. Note that only $D_{\mathrm{C}}$ involves $u$. The star type points are those with $D_{\mathrm{I}}<0$, which from (B.11), (B.12) has $\alpha_{\text {star }}=1 / 2$, implying that $\alpha_{\text {lemon }}+$ $\alpha_{\text {monstar }}=1 / 2$. In the following, it is convenient to change the $\phi_{i}$ angles to $\phi_{\mathrm{L}}=3 \phi_{1}+\phi_{2}, \phi_{\mathrm{C}}=\phi_{1}+$ $\phi_{2}-2 \phi_{3}, \phi=\phi_{3} / 2$, which may be done with jacobian 1 . Out of the original $\phi_{i}$ variables, $D_{\mathrm{LW}}$ now depends only on $\phi_{\mathrm{L}}, D_{\mathrm{CW}}$ only on $\phi_{\mathrm{C}}$.

The total fraction of lemon type is found using the fact that $D_{\mathrm{L}}<0$ for lemons; $D_{\mathrm{LW}}$ in (B.12) is independent of $r, u, \phi$, and $\phi_{\mathrm{C}}$, so integrating these from (B.11) is easy, and gives

$$
\begin{aligned}
\alpha_{\text {lemon }}= & \frac{1}{\pi} \int_{0}^{\pi / 2} \mathrm{~d} \theta \int_{0}^{2 \pi} \mathrm{d} \phi_{\mathrm{L}} \sin 2 \theta|\cos 2 \theta| \Theta\left(-D_{\mathrm{LW}}\right) \\
= & \frac{18}{100}+\frac{2}{\pi} \int_{\arccos (3 / \sqrt{10})}^{\pi / 4} \mathrm{~d} \theta \sin 2 \theta \cos 2 \theta \\
& \times \arccos \left(\frac{15-28 \cos 2 \theta+11 \cos 4 \theta}{4 \sin 2 \theta(1+\cos 2 \theta)}\right) \\
= & \frac{18}{100}+\frac{1}{\pi} \int_{0}^{4 / 5} \mathrm{~d} t t \arccos \left(\frac{4-28 t+22 t^{2}}{4(1+t) \sqrt{1-t^{2}}}\right) \\
= & 0.44721 .
\end{aligned}
$$

In this derivation, the second line was reached by integrating $\phi_{\mathrm{L}}$, the third line by substituting $\theta$ with $t=\cos 2 \theta$, the final line being a numerical 
integration. This derivation is similar to that for the fraction of lemon umbilic points in [25], Section 4.

The corresponding calculation for the elliptic (E) type, for which $D_{\mathrm{C}}>0$, is similar. After integrating out $r, \phi$ and $\phi_{\mathrm{L}}$, we have

$$
\begin{aligned}
\alpha_{\mathrm{E}}= & \frac{6}{\pi} \int_{0}^{\pi / 2} \mathrm{~d} \theta \int_{0}^{2 \pi} \mathrm{d} \phi_{\mathrm{C}} \int_{0}^{\infty} \mathrm{d} u \frac{u}{\left(1+u^{2}\right)^{4}} \\
& \times \sin 2 \theta|\cos 2 \theta| \Theta\left(D_{\mathrm{CW}}\right) \\
= & \frac{3}{2 \pi} \int_{0}^{\pi} \mathrm{d} \theta^{\prime} \int_{0}^{2 \pi} \mathrm{d} \phi_{\mathrm{C}} \int_{0}^{\infty} \mathrm{d} v \frac{\sin \theta^{\prime}\left|\cos \theta^{\prime}\right|}{(1+v)^{4}} \\
& \times \Theta\left(1-\sin ^{2} \theta^{\prime}-2 v\left(1-\cos \phi_{\mathrm{C}} \sin \theta^{\prime}\right)\right),
\end{aligned}
$$

where $v=u^{2}, \theta^{\prime}=2 \theta$. Therefore, in $\theta^{\prime}$, the integrand is symmetric about $\pi / 2$, that is, in index (from the form of $D_{\mathrm{IW}}$ ), so $\alpha_{\mathrm{E} \text {,star }}=\alpha_{\mathrm{E} \text {,lemon }}+$

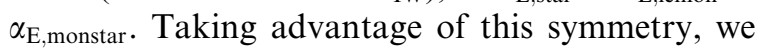
can substitute $s=\sin \theta^{\prime}$, and integrate $v$ :

$$
\begin{aligned}
\alpha_{\mathrm{E}}= & \frac{3}{\pi} \int_{0}^{1} \mathrm{~d} s \int_{0}^{2 \pi} \mathrm{d} \phi_{\mathrm{C}} \frac{s}{3} \\
& \times\left(1-\frac{8\left(1-s \cos \phi_{\mathrm{C}}\right)^{3}}{\left(3-s^{2}-2 s \cos \phi_{\mathrm{C}}\right)^{3}}\right)=\frac{1}{2},
\end{aligned}
$$

where the final integration is straightforward. This result implies that $1 / 2=\alpha_{\mathrm{E}}=\alpha_{\mathrm{H}}=2 \alpha_{\mathrm{E}, \mathrm{star}}$, etc.

The final density calculation is that of the elliptic lemons; although the total fraction of elliptic lemons and monstars is half, they are not necessarily in the same proportion as they are overall. Therefore, after integrating out $r$ and $\phi$ from (B.11)

$$
\begin{aligned}
\alpha_{\mathrm{E}, \text { lemon }}= & \frac{3}{\pi^{2}} \int_{0}^{\pi / 2} \mathrm{~d} \theta \int_{0}^{2 \pi} \mathrm{d} \phi_{\mathrm{L}} \int_{0}^{2 \pi} \mathrm{d} \phi_{\mathrm{C}} \\
& \times \int_{0}^{\infty} \mathrm{d} u \frac{u}{\left(1+u^{2}\right)^{4}} \sin 2 \theta|\cos 2 \theta| \\
& \times \Theta\left(D_{\mathrm{CW}}\right) \Theta\left(-D_{\mathrm{LW}}\right) \\
= & 0.23481
\end{aligned}
$$

where the final numerical result is arrived at from transformations similar to those used for $\alpha_{\text {lemon }}, \alpha_{\mathrm{E}}$, and the other fractions may be derived from those found here.

We now turn our attention to $\mathrm{L}$ lines, whose average density, by stationarity and ergodicity, can be found from Eq. (37), giving

$d_{\mathrm{L}}=\left\langle\delta\left(S_{3}\right)\left|\nabla S_{3}\right|\right\rangle$.

Now, $\mathbf{p}$ and $\mathbf{q}$ may be transformed to polar coordinates as before, and integrate $\theta_{0}$, giving

$$
\begin{aligned}
d_{\mathrm{L}}= & \frac{8}{\pi} \int_{0}^{\infty} \mathrm{d} p \int_{0}^{\infty} \mathrm{d} q \int_{0}^{2 \pi} \mathrm{d} \theta p q \delta(2 p q \sin \theta) \\
& \times \exp \left(-2\left(p^{2}+q^{2}\right)\right) \\
& \times \int \mathrm{d}^{4} \nabla \mathbf{p} \mathrm{d}^{4} \nabla \mathbf{q} p(\nabla \mathbf{p}, \nabla \mathbf{q})\left|\nabla S_{3}\right| \\
= & \frac{8}{\pi} \int_{0}^{\infty} \mathrm{d} p \int_{0}^{\infty} \mathrm{d} q \exp \left(-2\left(p^{2}+q^{2}\right)\right) \\
& \times \int \mathrm{d}^{4} \nabla \mathbf{p} \mathrm{d}^{4} \nabla \mathbf{q} p(\nabla \mathbf{p}, \nabla \mathbf{q})\left|\nabla S_{3}\right| .
\end{aligned}
$$

Transforming to polars $(p, q) \rightarrow(U, \phi)$, and using the fact that both $\mathbf{p}, \mathbf{q}$ are in the same direction (relabelled as the $x$-direction), $\left|\nabla S_{3}\right|$ becomes

$2 U \sqrt{c^{2}\left(\nabla q_{y}\right)^{2}+s^{2}\left(\nabla p_{y}\right)^{2}-2 c s \nabla p_{y} \cdot \nabla q_{y}}$,

where C, $s$ denote $\cos \phi$, $\sin \phi$, respectively. $\left|\nabla S_{3}\right|$ now only involves the derivatives of the $y$ components of $\mathbf{p}, \mathbf{q}$, so only these need to be integrated over. Writing these as a vector $\mathbf{V}=\sqrt{K_{2}} / 2$ $\left(p_{y, x}, p_{y, y}, q_{y, x}, q_{y, y}\right)$, and writing $\left|\nabla S_{3}\right|$ as a quadratic form $T K_{2} / 2|\mathbf{V} \cdot \mathbf{\Xi} \cdot \mathbf{V}|^{1 / 2}$, the integral becomes

$$
\begin{aligned}
d_{\mathrm{L}}= & \frac{4 \sqrt{K_{2} / 2}}{\pi^{3}} \int_{0}^{\infty} \mathrm{d} U U^{2} \exp \left(-2 U^{2}\right) \\
& \times \int_{0}^{\infty} \mathrm{d} \phi \int \mathrm{d}^{4} \mathbf{V}|\mathbf{V} \cdot \boldsymbol{\Xi} \cdot \mathbf{V}| \exp \left(-V^{2}\right)
\end{aligned}
$$

As before, $\mathbf{V}$ can be orthogonally transformed to a basis in which $\boldsymbol{\Xi}$ is diagonal, and therefore can easily be integrated, with the result

$d_{\mathrm{L}}=\frac{\pi}{4} \sqrt{\frac{K_{2}}{2}}$. 
Similar methods are employed to show that the corresponding density of $\mathrm{L}$ lines crossing a straight line $d_{\mathrm{s}, 1}$, starting from (38), is

$d_{\mathrm{L}, 1}=\frac{1}{2} \sqrt{\frac{K_{2}}{2}}$.

\section{References}

[1] J.F. Nye, Proc. R. Soc. London A 389 (1983) 279.

[2] J.F. Nye, Proc. R. Soc. London A 387 (1983) 105.

[3] J.F. Nye, Natural focusing and fine structure of light: caustics and wave dislocations, Institute of Physics Publishing, Bristol, 1999.

[4] J.F. Nye, J.V. Hajnal, Proc. R. Soc. London A 409 (1987) 21.

[5] J.V. Hajnal, Proc. R. Soc. London A 414 (1987) 433.

[6] J.V. Hajnal, Proc. R. Soc. London A 414 (1987) 447.

[7] M.V. Berry, M.R. Dennis, Proc. R. Soc. London A 457 (2001) 141.

[8] M.V. Berry, in: M.S. Soskin, M.V. Vasnetsov (Eds.), Singular Optics Optical Vortices: Fundamentals and Applications, SPIE, vol. 4403, 2001, p. 1.

[9] I. Freund, Opt. Lett. 26 (2001) 1996.

[10] A.I. Konukhov, L.A. Melnikov, J. Opt. B 3 (2001) S139.

[11] M.R. Dennis, Topological singularities in wave fields, Ph.D. Thesis, University of Bristol, 2002.

[12] I. Freund, Opt. Commun. 208 (2002) 223.

[13] O.V. Angelsky, I.I. Mokhun, A.I. Mokhun, M.S. Soskin, Phys. Rev. E 65 (2002) 036602.

[14] I. Freund, A.I. Mokhun, M.S. Soskin, O.V. Angelsky, I.I. Mokhun, Opt. Lett. 27 (2002) 545.

[15] O. Angelsky, A. Mokhun, I. Mokhun, M. Soskin, Opt. Commun. 207 (2002) 57.

[16] I. Freund, M.S. Soskin, A.I. Mokhun, Opt. Commun. 208 (2002) 57.

[17] J.F. Nye, M.V. Berry, Proc. R. Soc. London A 336 (1974) 165.

[18] M. Vasnetsov, K. Staliunas (Eds.), Optical Vortices, Nova Science, Commack, NY, 1999.

[19] M.V. Berry, M.R. Dennis, Proc. R. Soc. London A 456 (2000) 2059 (errata 456 3059).

[20] M.V. Berry, J. Phys. A: Math. Gen. 11 (1978) 27.

[21] B.I. Halperin, in: R. Balian, M. Kléman, J.-P. Poirier (Eds.), Les Houches Session XXV - Physics of Defects, North-Holland, Amsterdam, 1981, p. 813.

[22] I. Freund, Waves Rand. Med. 8 (1998) 119.

[23] M.R. Dennis, Local properties and statistics of phase singularities in generic wavefields, in: M.S. Soskin, M.V. Vasnetsov (Eds.), Singular Optics Optical Vortices: Fundamentals and Applications, SPIE, vol. 4403, 2001, p. 13.

[24] M.R. Dennis, J. Phys. A: Math. Gen. 34 (2001) L297.
[25] M.V. Berry, J.H. Hannay, J. Phys. A: Math. Gen. 10 (1977) 2083.

[26] I.R. Porteous, Geometric differentiation: for the intelligence of curves and surfaces, second ed., Cambridge University Press, Cambridge, MA, 2001.

[27] M.S. Longuet-Higgins, Trans. R. Soc. A 249 (1957) 321.

[28] M.S. Longuet-Higgins, Philos. Trans. R. Soc. A 250 (1957) 157.

[29] M.S. Longuet-Higgins, Proc. Cambridge Philos. Soc. 54 (1957) 439.

[30] R.D. Ray, J. Mar. Syst. 28 (2001) 1.

[31] A.S. Thorndike, C.R. Cooley, J.F. Nye, J. Phys. A: Math. Gen. 11 (1978) 1455.

[32] N.D. Mermin, Rev. Mod. Phys. 51 (1979) 591.

[33] R. Penrose, Ann. Hum. Gen. 42 (1979) 435.

[34] P.A.G. Scheuer, J.H. Hannay, P.J. Hargrave, Mon. Not. R. Astr. Soc. 180 (1977) 163.

[35] D. Brewster, Philos. Mag. 31 (1847) 444.

[36] G. Horvath, J. Gal, I. Pomozi, Naturwissenschaften 85 (1998) 333.

[37] M. Born, E. Wolf, Principles of Optics, Pergamon Press, Oxford, 1959.

[38] In the mathematical language of fibre bundles, the Poincaré sphere is the image of the three-sphere (with coordinates $\left.1 / \sqrt{S_{0}}\left(p_{x}, p_{y}, q_{x}, q_{y}\right)\right)$ under the Hopf map (Hopf fibration), and the Hopf circles are all those points which have different rectifying phases $\chi_{0}$, but are otherwise the same. The phase sphere is similar, but with Hopf circles parameterised by $\gamma_{0}$; the two different spheres are related by exchanging $p_{y}$ and $q_{x}$ in four-dimensional space.

[39] E. Majorana, Nuovo Cimento 9 (1932) 43.

[40] J.H. Hannay, J. Mod. Opt. 45 (1998) 1001.

[41] V.I. Arnold, Mathematical methods of classical mechanics, second ed., Springer, Berlin, 1989.

[42] M.V. Berry, in: G. Iooss, R.H.G. Helleman, R. Stora (Eds.), Les Houches Lecture Series Session XXXVI, North-Holland, Amsterdam, 1983, p. 171.

[43] M.V. Berry, J. Phys. A: Math. Gen. 8 (1975) 566.

[44] J.C. Dainty, Prog. Opt. 14 (1976) 1.

[45] J.W. Goodman, in: J.C. Dainty (Ed.), Laser speckle and related phenomena, Springer, Berlin, 1975, p. 9.

[46] R. Barakat, Opt. Acta 32 (1985) 295.

[47] D. Eliyahu, Phys. Rev. E 47 (1993) 2881.

[48] R. Barakat, J. Opt. Soc. Am. A 4 (1987) 1256.

[49] A.F. Fercher, P.F. Steeger, Opt. Acta 28 (1981) 443.

[50] D. Eliyahu, Phys. Rev. E 50 (1994) 2381.

[51] I. Freund, M. Wilkinson, J. Opt. Soc. Am. A 15 (1998) 2892.

[52] J.P. Hansen, I.R. McDonald, Academic Press, New York, 1986.

[53] M.R. Dennis, I. Freund, C point screening in random optical ellipse fields, Opt. Lett. (August) (2002), submitted.

[54] H. Solomon, Geometric Probability, SIAM, Arrowsmith, Bristol, 1978.

[55] M.R. Dennis, in preparation. 
[56] M. Abramowitz, I.A. Stegun (Eds.), Handbook of Mathematical Functions, Dover, New York, 1965.

[57] R. Zallen, H. Scher, Phys. Rev. B 4 (1971) 4471.
[58] A.V. Milovanov, G. Zimbardo, Phys. Rev. E 62 (2000) 250.

[59] M.B. Isichenko, Rev. Mod. Phys. 64 (1992) 961. 